Department of Policy and Planning Sciences

\author{
Discussion Paper Series
}

No.1367

The Evolution of Collective Choice Under Majority Rule

by

Akira OKADA, Ryoji SAWA

January 2020

Revised February 2021

UNIVERSITY OF TSUKUBA

Tsukuba, Ibaraki 305-8573

JAPAN 


\title{
The Evolution of Collective Choice under Majority Rule*
}

\author{
Akira Okada ${ }^{\dagger}$ and Ryoji Sawa
}

February 24, 2021

\begin{abstract}
We consider a dynamic process of collective choice under majority rule in which a status quo policy evolves. The analysis is based on stochastic evolutionary game theory. The Condorcet winner is uniquely a long-run equilibrium for all (super-)majority rules. When the Condorcet winner does not exist, the long-run equilibria under all majority voting rules belong to the top cycle of policies under a simple majority. When the policy space is multidimensional and the voting quota is larger than the min-max quota, the long-run equilibrium belongs to the minmax set. Finally, the Borda winner appears as a long-run equilibrium under unanimity if voters' behavior is governed by a logit choice rule.
\end{abstract}

Keywords: Collective choice; Voting; Stochastic evolutionary game theory; Condorcet winner; Borda winner.

*The authors thank Munetomo Ando, Michael Bognanno, Yi-Chun Chen, Toshimasa Maruta, Jonathan Newton, Daisuke Oyama, Satoru Takahashi, Dai Zusai, and seminar participants at the Japanese Economic Association 2015 Fall Meeting, SAET 2017 Conference, Learning, Evolution, and Games 2018 Conference, 284th Queueing Theory Workshop of the Operations Research Society of Japan, Monash University, National University of Singapore, Nihon University Population Research Institute, Osaka University, Tohoku University, University of Aizu, Keio University, University of Melbourne, University of New South Wales, University of Sydney, and Yokohama National University for their helpful comments and suggestions. We acknowledge financial support from the Japan Society for the Promotion of Science: JSPS KAKENHI Grants-in-Aid for Scientific Research (A) No. 26245024 (Okada), Young Scientists (B) No. 15K17023 (Sawa), and Early-Career Scientists No. 18K12740 (Sawa), and from Nomura Foundation (Grants in Social Science) (Sawa). This paper was formerly circulated as "An evolutionary approach to social choice problems with q-quota rules".

†professor emeritus, Hitotsubashi University, 2-1 Naka, Kunitachi, Tokyo 186-8601, JAPAN. Email:aokada313@gmail.com.

†Department of Policy and Planning Sciences, University of Tsukuba, Tennoudai 1-1-1, Tsukuba, Ibaraki 3058573, JAPAN. E-mail: rsawa@sk.tsukuba.ac.jp. 


\section{Introduction}

We present a dynamic model of collective choice under majority rule in which a status quo policy evolves, challenged by a new policy in every period. A challenging policy is drawn randomly from a finite set of policies. If the challenging policy wins the majority, then it becomes the status quo policy in the next period. Otherwise, the current status quo policy remains in effect. In contrast to the traditional literature on legislative bargaining, beginning with Baron and Ferejohn (1989), our analysis is based on stochastic evolutionary game theory. Voters are boundedly rational in the sense that they are myopic, and they may make a mistake, probabilistically. A model of boundedly rational behavior is suitable when voters do not have perfect foresight about voting outcomes, owing to uncertainty and complexity in the political environment and incomplete policy descriptions. This study aims to characterize long-run equilibria in dynamic policy-making processes.

According to Baron (1996), many of the collective choices are continuous, rather than once-off. Decisions made today may become the status quo in future and, thus, affect future decisions (e.g., constitutional amendments, referendums, and national elections). Therefore, we focus on long-run, rather than short-run outcomes of collective choices. An example of our setting is dynamic legislative bargaining, where a policy becomes the status quo in the next period if it receives a qualified majority. Legislators may make myopic decisions if their main concern is to be elected in a coming election. We focus on the evolution of the public policies implemented in such environments.

This study relates the static solution concepts of social choice theory to a long-run equilibrium in a dynamic voting process. The Condorcet (1785) winner is a policy that defeats all other policies under a simple majority rule. It is the median of the players' ideal policies when the policy space is one-dimensional and voters' preferences are single peaked (Black 1948). However, when the policy space has two or more dimensions, the set of undefeated policies under the simple majority (called the majority core) may be empty without strong assumptions; see Plott (1967), Tullock (1967), and Davis et al. (1972) for early studies on this topic. Furthermore, McKelvey (1976, 1979) shows that when the majority core is empty, the "top cycle" (i.e., the set of policies that can be reached from any other policy through a finite chain of majority preferences) is the whole policy space. ${ }^{1}$ That is, any policy can be reached from any other through a process of pairwise majority comparisons. This result is often interpreted as fragility or instability in majority voting (some call it chaos). Riker (1980) writes: "the sum of our new sophistication is, therefore, that political outcomes truly are unpredictable in the long run."

Simpson (1969) proposed the min-max policy, which is weaker than the Condorcet winner. For a policy $a$, let $n(a)$ be the maximum number of voters who vote against $a$ by voting for a

\footnotetext{
${ }^{1}$ Plott (1967) provides the necessary and sufficient conditions for the non-empty majority core. McKelvey (1976) shows that under certain conditions, the top cycle is always the whole policy space when the majority core is empty.
} 
different policy. A min-max policy minimizes $n(a)$ for all policies, and has "the property that the maximum number of voters wishing to move in any direction is a minimum." (Simpson, 1969). The set of min-max policies is called the min-max set, and the min-max number of voters the minmax quota. ${ }^{2}$ A min-max policy always exists, and the Condorcet winner (if it exists) is a unique min-max policy (when preferences are strict).

We employ stochastic evolutionary game theory to characterize a long-run equilibrium. The theory examines the robustness of each policy when voters' behavior includes the possibility of rare mistakes. Here, we assume a stochastic choice rule for voters in which, with a small positive probability, they may make a mistake by choosing a suboptimal policy. Specifically, we assume voters' error probabilities are identical and independent across policies and individuals. The dynamic process with the aforementioned choice rule is called the best-response dynamics with mutations (BRM). This dynamic process of collective choice generates a (irreducible and aperiodic) Markov process with finite states. A policy is stochastically stable (or a long-run equilibrium) if the stationary distribution of the Markov process places a positive probability on it in the limit, such that the error probability vanishes. Intuitively, a stochastically stable policy is the most likely equilibrium when random perturbations to voters' behavior are small. The set of stochastically stable policies is independent of an initial status quo policy. Furthermore, it is known that a stochastically stable state exists under a general choice rule satisfying a regularity condition (Sandholm, 2010).

Our results are summarized as follows. First, we prove that the Condorcet winner (if any) is uniquely stochastically stable for every majority rule in which the majority quota $q$ varies from a simple majority to unanimity. When the Condorcet winner does not exist, every stochastically stable policy under every majority rule (including unanimity) belongs to the top cycle under a simple majority.

Second, even in settings with no Condorcet winner, we find conditions to further characterize long-run equilibria among policies in the top cycle. The long-run equilibria under each majority voting rule must belong to the min-max set when the majority quota $q$ is larger than the min-max quota, and the policy space is multidimensional and discretized sufficiently finely. This implies that stochastic evolutionary theory mitigates the indeterminacy problem of majority voting for multidimensional policy spaces. If the voting quota is less than the min-max quota, majority voting may cause the intransitivity of social choice (the paradox of voting). If the quota is greater than the min-max quota, we confront the indeterminacy problem - the core includes many policies. In particular, under unanimity, the core coincides with the set of Pareto efficient policies. Stochastic evolutionary theory shows that policies stay in the min-max set in the long run, which is much

\footnotetext{
${ }^{2}$ The min-max quota is the minimal majority size under which an undefeated policy exists. Caplin and Nalebuff (1988) show that when the policy space is multidimensional and the density of voters' ideal points is concave over its support, the min-max quota is less than $64 \%$ of voters.
} 
smaller than the set of Pareto efficient policies.

Third, to examine the influence of the voting behavior on long-run equilibria, we consider another probabilistic voting model: a logit rule in which voters' error probabilities are governed by the logit function. We prove that the Borda (1781) winner is the unique long-run equilibrium under the unanimity rule. This has a positive implication for the normative (and old) debate concerning the Condorcet winner versus the Borda winner: either solution can emerge as a long-run equilibrium of a dynamic unanimity voting process, depending on the voters' behavioral model.

We discuss several strands of the literature related to this paper. ${ }^{3}$ Our result provides an evolutionary approach to recent literature on legislative bargaining with an endogenous status quo policy, as proposed by Baron (1996); see Kalandrakis (2004), Battaglini and Coate (2007), Penn (2009), Anesi (2010), Duggan and Kalandrakis (2012), Anesi and Seidmann (2015), Diermeier et al. (2017), and Nunnari (2021), among others. Most studies in this literature employ the rational choice paradigm, which assumes voters maximize their long-term utility, have perfect foresight, and have perfect knowledge of others' preferences. Baron (1996) presents a (now standard) model of legislative bargaining with an endogenous status quo, where a legislator is selected randomly to propose a policy. In the unidimensional case, he characterizes a stationary Markov perfect equilibrium in which the outcome converges to the median's ideal point (the Condorcet winner). Duggan and Kalandrakis (2012) establish the existence of a stationary Markov perfect equilibrium in a multidimensional case with general preferences, and prove the convergence to the Condorcet winner under a weighted majority rule. In a three-person divide-the-dollar game under majority voting, Kalandrakis (2004) shows that the proposer eventually exploits the whole dollar in all periods, irrespective of the common discount factor. Nunnari (2021) extends the model of Kalandrakis (2004) to the one with a veto player and shows, both theoretically and experimentally, that there is always an equilibrium where the veto player eventually exploits the whole dollar. Anesi and Seidmann (2015) extend the result of Kalandrakis (2004) to an $n$-person divide-the-dollar game. They focus on a special class of no-delay stationary Markov perfect equilibria in which the first proposal is accepted and remains effective in all future rounds. In the case of a finite policy space, Anesi (2010) and Diermeier et al. (2017) characterize a von-Neumann and Morgenstern stable set as the limit set of a stationary Markov perfect equilibrium. Battaglini and Coate (2007) examine the efficiency of legislative bargaining as it relates to public spending and taxation. Penn (2009) considers a model of legislative bargaining with logit choice rules, where a proposal is chosen according to a fixed probability distribution, similarly to ours. She proves the existence and uniqueness of a value function that expresses voters' long-term preferences over policies, but she does not characterize

\footnotetext{
${ }^{3}$ For applications of stochastic evolutionary game theory in cooperative settings, see Agastya (1999), Jackson and Watts (2002), Arnold and Schwalbe (2002), Klaus et al. (2010), Newton (2012), Sawa (2014), Newton and Sawa (2015), Nax and Pradelski (2015), Boncinelli and Pin (2018), and Bilancini et al. (2020). See also Section 2 of Newton (2018).
} 
the long-run equilibrium.

Other related studies are those that consider evolutionary dynamics in voting settings; see Kanazawa (1998), Bendor et al. (2003), Fowler (2006), Conley et al. (2006), and Landi and Sodini (2012), for example. Their main concern is the paradox of voter turnout - people do vote even if the cost of voting is seemingly greater than the benefit of the chance of being pivotal. Kanazawa (1998) considers adaptive learning dynamics with voters who learn from the past correlations between their choices and the outcomes. Bendor et al. (2003) and Fowler (2006) consider evolutionary dynamics where voters adjust their aspiration levels that determine whether outcomes are satisfactory or not. Conley et al. (2006) adopt a model of preference evolution, where preferences associated with propensities to vote evolve. Landi and Sodini (2012) consider best response dynamics with conformist voters, i.e., voters prefer to do what others do. These studies offer some evolutionary model that can explain the paradox.

There are several papers that consider social dynamics in different contexts from ours. Lagunoff and Matsui (1995) consider dynamic processes under a class of decision mechanisms, including majority voting, that map players' yes/no strategies to their (mandatory) contribution for provision of a public good. Gomes and Jehiel (2005), Acemoglu et al. (2011), and Acemoglu et al. (2012) consider dynamic settings with farsighted players where a winning coalition chooses the next state in each period. It is shown that in general, the state that such dynamic processes converge to may or may not be Pareto efficient. Nax and Rigos (2016) and Wu (2016) consider dynamic settings where players endogenously make the choice of assortative mechanism under democratic processes, e.g. majority voting. Belloc and Bowles (2017) consider the simultaneous evolution of preferences and institutions, and Wu (2017) considers the evolution of character traits under different political institutions.

Besides legislative bargaining problems, evolutionary and learning models of collective choice can be applied to many social and economic problems. Among them, the theory of social contract is an important application. While there are many works which consider justice and social contract in the framework of evolutionary game theory (Binmore 1994 and 2005, Skyrms 1996 and Young 1993 and 1998, etc.), most works are restricted to the two-person case. Our evolutionary result on collective choice is an attempt to incorporate the notion of a coalition into the literature. Another interesting application is a dynamic opinion formation process. When players receive noisy private signals about a true state, gather their neighbors' opinions, and update their own views repeatedly, Golub and Jackson (2010) show that all players' opinions may converge to a truth under a naïve learning process. See Grabisch and Rusinowska (2020) for a survey on the recent developments.

To conclude the introduction, it may be useful to compare the rational choice approach and our evolutionary approach to the collective choice problem in three aspects. The first is the assumption of rationality. In the rational choice approach, voters are assumed to be rational, make no mistakes, 
and have perfect knowledge of others' preferences. In contrast, the evolutionary approach assumes that voters may make a mistake, with small probability, and does not rely on the assumption of common knowledge about others' preferences. In our view, both approaches are complementary, providing a better understanding of complex real-world voting processes.

The second aspect is related to the modelling. A standard model of legislative bargaining randomly selects a legislator to make a proposal in each round. In contrast, the proposal process in our evolutionary model (and in Penn (2009)) is probabilistic and exogenous. In reality, a proposal process is determined by institutional factors, including formal recognition protocols, negotiations over proposal rights, and social norms (e.g., seniority). To minimize the dependence on the details of the model, we take an institution-free approach: rather than formulating an institutional process explicitly, we assume a proposal occurs through an unknown random process. The set of long-run equilibria is invariant with respect to a probability distribution over proposals.

The third aspect is the sensitivity to model parameters. Legislative bargaining results are often sensitive to the model parameters, e.g. voting rules, cardinal utilities, discount factors, and a probability distribution when choosing a proposer. In contrast, the long-run equilibrium of our evolutionary model depends on a smaller set of parameters, such as ordinal preferences and voting rules, given a stochastic choice rule for voters.

The remainder of the paper is organized as follows. Section 2 presents the collective choice model. Section 3 formulates the dynamic policy-making process. Section 4 characterizes the longrun equilibrium under the BRM. Section 5 extends the analysis to the case of multidimensional policy spaces, and Section 6 considers the logit choice rule under unanimity. Section 7 concludes the paper. All proofs are given in the Appendix.

\section{The Collective Choice Model}

Let $N=\{1, \ldots, n\}$ be the set of players, and let $\mathscr{A}$ be a finite set of policies. Each player $i$ has a strict preference over $\mathscr{A}$ (indifference is not allowed). The utility function of player $i$ is a realvalued function $u_{i}: \mathscr{A} \rightarrow \mathbb{R}$. In what follows, $N, \mathscr{A}$, and $\left\{u_{i}\right\}_{i \in N}$ are fixed. Players collectively choose a policy under majority voting. We consider a class of majority voting rules with different quotas. Here, a quota is denoted as $q \in\{1, \ldots, n\}$, which is the minimum number of votes required for a challenging policy to win against a status quo policy. The quota for a simple majority is given by $q=\frac{n+1}{2}$ for odd $n$, and by $q=\frac{n}{2}+1$ for even $n$. For our analysis, we introduce the following notation:

$$
\underline{q}= \begin{cases}\frac{n+1}{2} & \text { for odd } n \\ \frac{n}{2} & \text { for even } n\end{cases}
$$


Briefly, $\underline{q}$ is the largest integer not greater than $(n+1) / 2$. The quota for a simple majority is equal to $\underline{q}$ for odd $n$, and to $\underline{q}+1$ for even $n$. When $q=n$, the majority voting is unanimity. We focus on the case in which the quota $q$ is greater than or equal to $\underline{q}$. In what follows, a majority voting rule with a $q$-quota is referred to simply as a $q$-majority.

For any pair of policies $a, a^{\prime} \in \mathscr{A}$, let $N\left(a, a^{\prime}\right)$ denote the set of players who prefer $a^{\prime}$ to $a$, and $n\left(a, a^{\prime}\right)$ be the number of such players. We say that $a^{\prime}$ defeats $a$ under a $q$-majority if $n\left(a, a^{\prime}\right) \geq q$, and that $a$ is undefeated under a $q$-majority if it is not defeated by any other policy under the $q$-majority.

Given a quota $q$, the $q$-majority core is the set of undefeated policies under a $q$-majority. We denote the $q$-majority core by $C(q)$, which may be empty. For two policies $a, a^{\prime} \in \mathscr{A}$ and two quotas $q$ and $q^{\prime}$, with $q<q^{\prime}, a^{\prime}$ defeats $a$ under a $q$-majority if $a^{\prime}$ defeats $a$ under the $q^{\prime}$-majority; thus, $C(q) \subset C\left(q^{\prime}\right)$. The $q$-majority core expands as $q$ increases. The $n$-majority core $C(n)$ is equal to the set of Pareto efficient policies. Define

$$
\bar{n}(a)=\max _{a^{\prime} \in \mathscr{A} \backslash\{a\}} n\left(a, a^{\prime}\right)
$$

which is the maximum number of voters who prefer some policy $a^{\prime}$ to $a$. A policy $a$ is defeated by some other policy under a $q$-majority if and only if $\bar{n}(a) \geq q$. Thus, the $q$-majority core $C(q)$ is equal to the set $\{a \in \mathscr{A}: \bar{n}(a)<q\}$.

A Condorcet winner, denoted as $a^{C W}$, is a policy that defeats any other policy under a simple majority. By definition, $a^{C W} \in \mathscr{A}$ is a Condorcet winner if $n\left(a, a^{C W}\right)>n / 2$, for all $a \neq a^{C W}$. A Condorcet winner $a^{C W}$ (if any) is unique and belongs to the simple majority core. Under the assumption of strict preferences, it holds that $n\left(a, a^{\prime}\right)+n\left(a^{\prime}, a\right)=n$, for $a \neq a^{\prime}$. Thus, a policy $a \in \mathscr{A}$ is a Condorcet winner if and only if $\bar{n}(a)<n / 2$. For any $a \neq a^{C W}, n\left(a, a^{C W}\right)>n / 2$, and thus $\bar{n}(a)>n / 2$. Therefore, the Condorcet winner $a^{C W}$ minimizes $\bar{n}(a)$ for all policies $a \in \mathscr{A}$.

The literature on social choice theory has proposed two generalized notions of the Condorcet winner: the top cycle (Miller, 1977), and the min-max set (Simpson, 1969).

A policy $a \in \mathscr{A}$ is said to indirectly defeat $b \in \mathscr{A}$ under a $q$-majority, denoted as $a \succeq_{q}^{*} b$, if there exists a sequence $\left(a_{1}, \ldots, a_{m}\right)$ of policies with $a_{1}=b$ and $a_{m}=a$, such that $a_{i+1}$ defeats $a_{i}$ for every $i=1, \cdots, m-1$. The top cycle under a $q$-majority is a set of policies, each member of which indirectly defeats every other policy in $\mathscr{A}$. Let $\mathscr{A}_{q}^{t c}$ denote the top cycle under a $q$-majority. Formally, $\mathscr{A}_{q}^{t c}$ is defined as the set $\left\{a \in \mathscr{A}: \forall a^{\prime} \in \mathscr{A}, a^{\prime} \neq a, a \succeq_{q}^{*} a^{\prime}\right\}$. There are noteworthy properties of the top cycle. The Condorcet winner (if any) is the top cycle under a simple majority. The top cycle $\mathscr{A}_{q}^{t c}$ exists for every $q \leq \underline{q}$, but may be empty for $q>\underline{q}$. If the $q$-majority core $C(q)$ is nonempty, then the top cycle $\mathscr{A}_{q}^{t c}$ must be a subset of the core. ${ }^{4}$ Furthermore, $\mathscr{A}_{q}^{t c}$ is empty

\footnotetext{
${ }^{4}$ Any policy not in $C(q)$ does not indirectly defeat policies in $C(q)$ and, thus, is not in $\mathscr{A}_{q}^{t c}$.
} 
if $C(q)$ contains more than one policy. ${ }^{5}$ In addition, $C(q)$ is empty if the top cycle $\mathscr{A}_{q}^{t c}$ contains more than one policy. ${ }^{6}$ The top cycle plays a role only when the $q$-majority core is empty. ${ }^{7}$ Miller (1977, Theorem 7) shows the following characterization of the top cycle under a $q$-majority. The top cycle $\mathscr{A}_{q}^{t c}$ is the minimal undefeated set: (i) no policy in $\mathscr{A}_{q}^{t c}$ is defeated by any policy not in $\mathscr{A}_{q}^{t c}$ under the $\underline{q}$-majority; and (ii) no proper subset of $\mathscr{A}_{q}^{t c}$ satisfies (i).

A min-max policy is a policy that minimizes $\bar{n}(a)$ over $\mathscr{A}$. The minimum number of $\bar{n}(a)$ for $a \in \mathscr{A}$ is called the min-max quota, denoted by $\bar{n}$. It is the maximum number of voters who prefer some policy to a min-max policy. The set of min-max policies is called the min-max set, and is denoted as $\mathscr{A}^{m m}$. The min-max set $\mathscr{A}^{m m}$ is always nonempty. If the Condorcet winner $a^{C W}$ exists, then $a^{C W}$ is a unique min-max policy. The relationship of the min-max set $\mathscr{A}^{m m}$ to the $q$ majority core $C(q)$ is as follows. By definition, a min-max policy is undefeated under a $q$-majority rule if $q>\bar{n}$. Thus, $\mathscr{A}^{m m} \subseteq C(q)$ if $q>\bar{n}$. When $q=\bar{n}+1$, every policy $a$ in $C(\bar{n}+1)$ satisfies $\bar{n}(a)<\bar{n}+1$. From the definition of $\bar{n}$, it must hold that $\bar{n}(a)=\bar{n}$ for every policy $a$ in $C(\bar{n}+1)$. Thus, $\mathscr{A}^{m m}=C(q)$ if $q=\bar{n}+1$. When $q \leq \bar{n}, \mathscr{A}^{m m} \neq C(q)=\emptyset$.

A myopic stable set (MSS) is a general solution concept for cooperative settings (Demuynck et al. 2019). In our context, it is the set of all voting cycles under a given $q$-majority. Formally, a set $M S S_{q} \subseteq \mathscr{A}$ is a myopic stable set under a $q$-majority if it satisfies the three conditions: (i) for all $a \in M S S_{q}, a^{\prime} \in M S S_{q}$ if $a^{\prime} \succeq_{q}^{*} a$, (ii) for all $a \in \mathscr{A} \backslash M S S_{q}$, there is $a^{\prime} \in M S S_{q}$ such that $a^{\prime} \succeq_{q}^{*} a$, and (iii) $M S S_{q}$ is the minimal set satisfying (i) and (ii). The first condition implies that a $M S S_{q}$ is closed under better replies of coalitions of size $q$. The second condition implies that for any policy outside $M S S_{q}$, there is a sequence of (coalitional) better replies that leads to $M S S_{q}$. For every $q \in\{\underline{q}, \ldots, n\}, M S S_{q}$ exists and is unique. For a $\underline{q}$-majority, $M S S_{q}=\mathscr{A}_{\underline{q}}^{t c}$.

The next example illustrates the above concepts.

Example 1. Consider a collective choice problem with 25 voters and 4 policies. $q=\frac{25+1}{2}=13$. The voters' preferences are summarized in Table 1. Each cell in the right matrix gives the total number of voters who prefer a row policy to a column policy. For example, 25 voters prefer $a_{2}$ to $a_{3}$. The Condorcet winner is $a_{1}$, because it wins 13 votes against any other policy. The $q$-majority core is $\left\{a_{1}\right\}$ for $q=13$, and is $\left\{a_{1}, a_{2}\right\}$ for all $q \geq 14$. Policy $a_{2}$ is in the $q$-majority core for $q \geq 14$ since it does not lose 14 votes against any policy (to see this, check the column of ' $a_{2}$ ', which shows that $a_{2}$ loses at most 13 votes). The top cycle is $\left\{a_{1}\right\}$ for $q=13$, and is empty for all $q \geq 14$. The min-max set is $\left\{a_{1}\right\}$, and the min-max quota is $\bar{n}=12$ since the number of voters who prefer another policy to the Condorcet winner $a_{1}$ is at most 12 . The myopic stable set coincides with the $q$-majority core for every $q \geq 13$.

\footnotetext{
${ }^{5}$ Consider $a, a^{\prime} \in C(q)$. Policies $a$ and $a^{\prime}$ are not in the top cycle either, because $a \nsucceq_{q}^{*} a^{\prime}$ and $a^{\prime} \Varangle_{q}^{*} a$.

${ }^{6}$ If $a, a^{\prime} \in \mathscr{A}_{q}^{t c}$, then $a \succeq_{q}^{*} a^{\prime}$ and $a^{\prime} \succeq_{q}^{*} a$, which implies that $a$ and $a^{\prime}$ can be defeated. Because all policies outside $\mathscr{A}_{q}^{t c}$ are defeated, every policy is defeated. That is, the $q$-majority core is empty.

${ }^{7}$ If both concepts are nonempty, then they reduce to a common single element.
} 
Table 1: Preference and voting matrices of 25 voters and four policies

\begin{tabular}{|c|c|}
\hline Preferences & \# of players \\
\hline$a_{1} \succ a_{2} \succ a_{3} \succ a_{4}$ & 13 \\
\hline$a_{2} \succ a_{4} \succ a_{3} \succ a_{1}$ & 12 \\
\hline
\end{tabular}

\begin{tabular}{|c|c|c|c|c|}
\hline & $a_{1}$ & $a_{2}$ & $a_{3}$ & $a_{4}$ \\
\hline$a_{1}$ & - & 13 & 13 & 13 \\
\hline$a_{2}$ & 12 & - & 25 & 25 \\
\hline$a_{3}$ & 12 & 0 & - & 13 \\
\hline$a_{4}$ & 12 & 0 & 12 & - \\
\hline
\end{tabular}

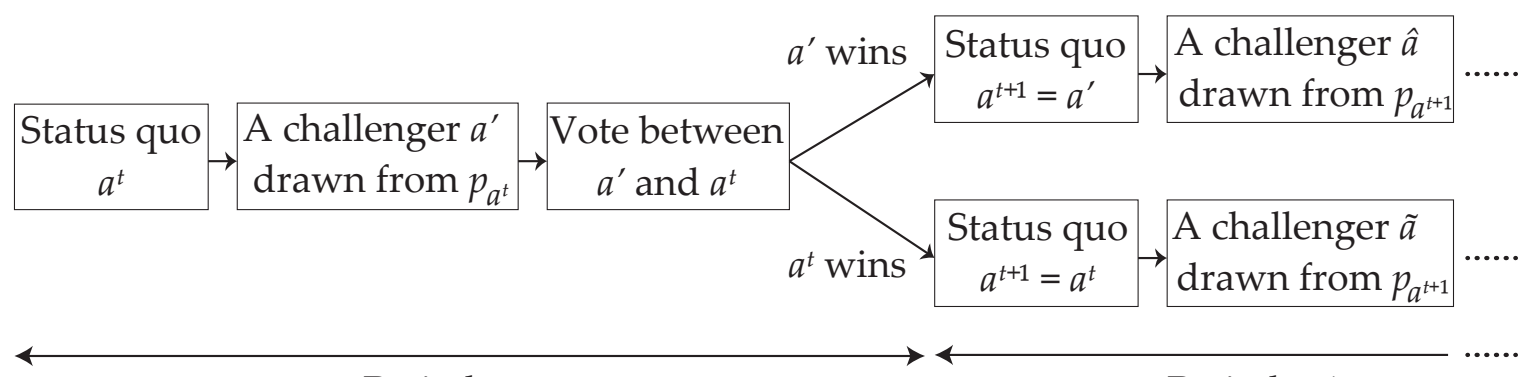

Period $t$

Period $t+1$

Figure 1: Path of play

\section{A Dynamic Policy-Making Process}

We consider a dynamic political process in which players vote recurrently using pairwise comparisons under a $q$-majority. The status quo policy in period $t$ is denoted as $a^{t} \in \mathscr{A}$. A policy $a \in \mathscr{A}$ is drawn against the status quo policy $a^{t}$ according to a (predetermined) probability distribution $p_{a^{t}}$. Every policy has a positive chance of being selected as a challenging policy. ${ }^{8}$ All players vote simultaneously between $a$ and $a^{t}$, according to their choice rules, which are defined below. If the challenging policy $a$ wins at least $q$ votes, it will become the status quo policy in the next period. Otherwise, $a^{t}$ remains as the status quo policy. Figure 1 illustrates the process.

We consider a probabilistic voting behavior that has been well studied in the literature (e.g., see Coughlin (1992), Banks and Duggan (2005), Schofield (2005), McKelvey and Patty (2006), and Penn (2009)). Specifically, we assume every player might, with a small probability, vote for a suboptimal policy. Such stochastic behavior can occur for several reasons. First, players are not perfectly rational, and thus may choose a suboptimal policy by mistake. Second, players are uncertain about the outcomes of policies, owing to random shocks to the political environment. Third, incomplete descriptions of policies may result in stochastic voting. In what follows, we interpret stochastic voting as occurring owing to voters' mistakes.

For all $a, a^{\prime} \in \mathscr{A}$, let $\Psi_{i}\left(a, a^{\prime}\right)$ denote the probability that player $i$ votes for $a^{\prime}$ against $a$. When

\footnotetext{
${ }^{8}$ The probability distribution over policies can be arbitrary. Our results are not affected in any critical way by the choice of distribution, as long as each policy has a positive probability of being selected.
} 
player $i$ makes no error, $\Psi_{i}\left(a, a^{\prime}\right)$ is given by ${ }^{9}$

$$
\Psi_{i}^{0}\left(a, a^{\prime}\right)= \begin{cases}1 & \text { if } u_{i}\left(a^{\prime}\right)>u_{i}(a) \\ 0 & \text { if } u_{i}\left(a^{\prime}\right) \leq u_{i}(a)\end{cases}
$$

Each player votes for the best policy with probability one. Under the possibility of making mistakes, each player $i$ 's choice is given by the following: for all $a, a^{\prime} \in \mathscr{A}$,

$$
\Psi_{i}^{\varepsilon}\left(a, a^{\prime}\right)= \begin{cases}1-\varepsilon & \text { if } u_{i}\left(a^{\prime}\right)>u_{i}(a) \\ \varepsilon & \text { if } u_{i}\left(a^{\prime}\right) \leq u_{i}(a)\end{cases}
$$

where $\varepsilon>0$ is the probability of making a mistake. Each player votes for the optimal policy with probability $1-\varepsilon$, and votes for a suboptimal policy with probability $\varepsilon$. This stochastic choice rule is called the BRM. The formal transition probabilities of the process can be found in Appendix A.

The aim of our analysis is to characterize the long-run equilibrium of a dynamic process of majority voting when $\varepsilon$ is sufficiently small. The perturbed process with $\varepsilon>0$ is a Markov chain with a unique stationary distribution, denoted as $\pi_{\varepsilon}^{q}$. Let $\pi_{\varepsilon}^{q}(a)$ denote the probability that $\pi_{\varepsilon}^{q}$ places on policy $a \in \mathscr{A}$. Let $\pi_{\varepsilon}^{q}\left(\mathscr{A}_{1}\right)=\sum_{a \in \mathscr{A}_{1}} \pi_{\varepsilon}^{q}(a)$ for a subset $\mathscr{A}_{1} \subseteq \mathscr{A}$. Then, the players' behavior is summarized asymptotically by the stationary distribution $-\pi_{\varepsilon}^{q}(a)$ represents the fraction of time in which policy $a$ is enacted over a long time horizon. In what follows, we refer to a policy in $\mathscr{A}$ a state of the process, if no confusion arises.

Definition. A state $z \in \mathscr{A}$ is stochastically stable under a q-majority if

$$
\lim _{\varepsilon \rightarrow 0} \pi_{\varepsilon}^{q}(z)>0 \text {. }
$$

In general, a stochastically stable state is the policy most likely to be enacted in the long term when voters' behavior is probabilistic, but the mistake rate is small. Theorem 3.16 of Demuynck et al. (2019) show that the set of recurrent states of the unperturbed process $(\varepsilon=0)$ coincides with $M S S_{q}$. This means that all stochastically stable policies under a $q$-majority must be in $M S S_{q}$ (see Theorem A.1 and its implication).

We provide the computation method, established in the literature on stochastic evolutionary game theory, for a stochastically stable state in Appendix A. Here, we present the essence of the method. For a policy $z \in \mathscr{A}$, the notion of a $z$-tree plays a critical role in the computation. From the standard definition in graph theory, a (directed) tree on $\mathscr{A}$ is a set of $|\mathscr{A}|-1$ transitions (directed edges) with three properties: (i) exactly one policy, called the root of the tree, has no exiting transition; (ii) all policies but the root have exactly one exiting transition; and (iii) for any policy

\footnotetext{
${ }^{9}$ Players prefer the status quo $a$ if $u(a)=u\left(a^{\prime}\right)$. Note that the assumption of strict preferences implies that $u_{i}(a)=$ $u_{i}\left(a^{\prime}\right)$ if and only if $a=a^{\prime}$. Our result is not affected by the choice in this case.
} 


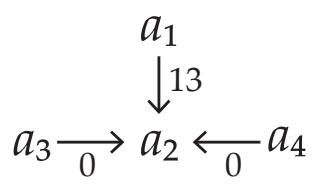

(a) $a_{2}$-tree

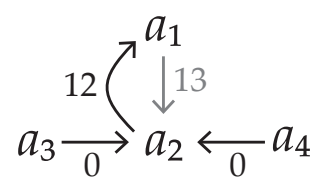

(b) $a_{1}$-tree

Figure 2: A non- $\underline{q}$-majority $a_{2}$-tree and a $\underline{q}$-majority $a_{1}$-tree

there is a unique path to the root. ${ }^{10}$ If a tree has a root $z \in \mathscr{A}$, we call it a $z$-tree. For each transition of the $z$-tree, we determine the minimum number of mistakes needed for the transition to occur. This number is called the transition cost, and is a measure of how unlikely the transition is to occur. The cost of a $z$-tree is defined as the sum of all transition costs within the tree. A policy $z \in \mathscr{A}$ is stochastically stable if and only if it has a $z$-tree with the smallest cost over $\mathscr{A}$.

\section{The Long-Run Equilibrium: Condorcet Winner and Top- Cycle}

In this section, we characterize stochastically stable policies under a $q$-majority. The analysis is restricted to an interesting case of $q \in\{q, \ldots, n\}$. It is useful to introduce the notion of a $q$ majority tree ${ }^{11}$ A $q$-majority tree is a tree on the policy space $\mathscr{A}$, where each transition has zero cost under the $q$-majority. That is, $z$-tree for $z \in \mathscr{A}$, say $\tau_{z}$, is a $q$-majority tree if $n\left(a^{\prime}, a^{\prime \prime}\right) \geq q$, for all $\left(a^{\prime}, a^{\prime \prime}\right) \in \tau_{z}$. Every transition of a $q$-majority tree is preferred by at least $q$ voters. The root of a $q$-majority tree can be reached from any other policy, with no mistakes, by repeating the $q$-majority voting. We focus on $q$-majority trees with $q=\underline{q}$. The next example illustrates $\underline{q}$-majority trees.

Example 2. Recall the collective choice problem in Example 1, where 25 voters choose from 4 policies $\left\{a_{1}, \ldots, a_{4}\right\}$. Recall also that $\underline{q}=13$, and $a_{1}$ is the Condorcet winner. Figure 2(a) shows an $a_{2}$-tree, and Figure 2(b) shows an $a_{1}$-tree. An arrow from $a_{j}$ to $a_{h}$ denotes a transition from $a_{j}$ to $a_{h}$. A number associated with an arrow indicates the number of voters who oppose the transition.

The $a_{2}$-tree in Figure 2(a) is not a $\underline{q}$-majority tree because the edge $\left(a_{1}, a_{2}\right)$ has $n\left(a_{1}, a_{2}\right)=$ $12<\underline{q}$. The $a_{1}$-tree in Figure 2(b), obtained by replacing $\left(a_{1}, a_{2}\right)$ with $\left(a_{2}, a_{1}\right)$ in Figure 2(a), is a $\underline{q}$-majority tree. The number of opposing voters is less than $\underline{q}$ for every edge in Figure 2(b).

The following lemma provides a necessary condition for a policy to be stochastically stable.

Lemma 1. Let $q \geq \underline{q}$. Every stochastically stable policy $z^{*} \in \mathscr{A}$ under a $q$-majority must be the root of some q-majority tree.

\footnotetext{
${ }^{10}$ For example, Figure 2(a) shows a tree with four policies.

${ }^{11}$ Our notion of a majority tree is similar to that of a complete path in Miller (1977).
} 
Lemma 1 implies that, for every $q$-majority rule, a policy $z^{*}$ is stochastically stable only if $z^{*}$ can be reached from any policy $z$ under the $q$-majority rule through a sequence of pairwise voting. Let $q \in\{\underline{q}, \ldots, n\}$. Intuitively, suppose $z^{*}$ is not the root of any $q$-majority tree. Then, there is some policy, say $z$, from which $z^{*}$ cannot be reached, with no mistakes, under the $q$-majority using any sequence of pairwise voting. It can be shown that $z$ is more likely to be enacted than $z^{*}$. To see this, choose a $z^{*}$-tree that minimizes the cost among all possible $z^{*}$-trees. The chosen $z^{*}$-tree must have a transition, say $\left(z, z^{\prime}\right)$, that is not supported by the majority, i.e. $n\left(z, z^{\prime}\right)<\underline{q}$. Its transition cost is $q-n\left(z, z^{\prime}\right)>q-\underline{q}$. Note that the transition cost of $\left(z, z^{*}\right)$ is greater than or equal to that of $\left(z, z^{\prime}\right)$; that is, $q-n\left(z, z^{*}\right) \geq q-n\left(z, z^{\prime}\right)$. Otherwise, we can form a $z^{*}$-tree with a strictly smaller cost by replacing $\left(z, z^{\prime}\right)$ with $\left(z, z^{*}\right)$. We construct a $z$-tree by replacing $\left(z, z^{\prime}\right)$ with $\left(z^{*}, z\right)$. The transition cost of $\left(z^{*}, z\right)$ is at most $q-\underline{q}$. Then, the cost of the $z$-tree is strictly smaller than that of the $z^{*}$-tree, which contradicts that $z^{*}$ is stochastically stable under a $q$-majority.

We can now state our main result. The proof is given in Appendix B.

Theorem 1. The Condorcet winner is uniquely stochastically stable under the BRM for all $q \geq$ $\underline{q}^{1213}$

The theorem is straightforward for the simple majority voting, where the Condorcet winner can be reached, without error, from any other policy under the simple majority. In contrast, every other policy can be reached from the Condorcet winner only when at least one voter makes a mistake. Thus, the Condorcet winner is the unique long-run equilibrium under a simple majority.

An important finding of Theorem 1 is that the Condorcet winner is uniquely stochastically stable for all majority rules, including unanimity. When $q$ is a super-majority rule, the Condorcet winner may not always win the super majority. For example, the core $C(q)$ may include policies other than the Condorcet winner; that is, many policies may be undefeated under the $q$-majority. If enacted, such a policy remains as the status quo, as long as voters make no errors. However, the Condorcet winner $a^{C W}$ is the only policy to be a long-run equilibrium, even under a super majority rule. Intuitively, any $a$-tree for $a \neq a^{C W}$ has a transition exiting from $a^{C W}$. The transition is not preferred by a majority and, thus, it is impossible for any $a \neq a^{C W}$ to be the root of a $\underline{q}$-majority tree. Therefore, by Lemma 1, any policy other than the Condorcet winner cannot be a long-run equilibrium under any $q$-majority rule.

Theorem 1 implies the following. In the literature on social choice theory, the core has frequently been studied as a set of stable majority voting outcomes. The core expands as the voting

\footnotetext{
${ }^{12}$ When the Condorcet winner does not exist, we can prove a weak version of Theorem 1: When $n$ is even, a weak Condorcet winner $a^{*}$ satisfying $n\left(a^{\prime}, a^{*}\right) \geq n / 2$, for all $a^{\prime} \neq a^{*}$, is stochastically stable under every $q$-majority.

${ }^{13}$ If we allow $q<q$, such majority voting rules actually describe minority rules in which a challenging policy is selected if a minority group of players prefer it. The Condorcet winner is uniquely stochastically stable even under such minority rules as long as $q>\bar{n}$.
} 
Probability distribution on the Condorcet winner and $\mathrm{a}_{2}$

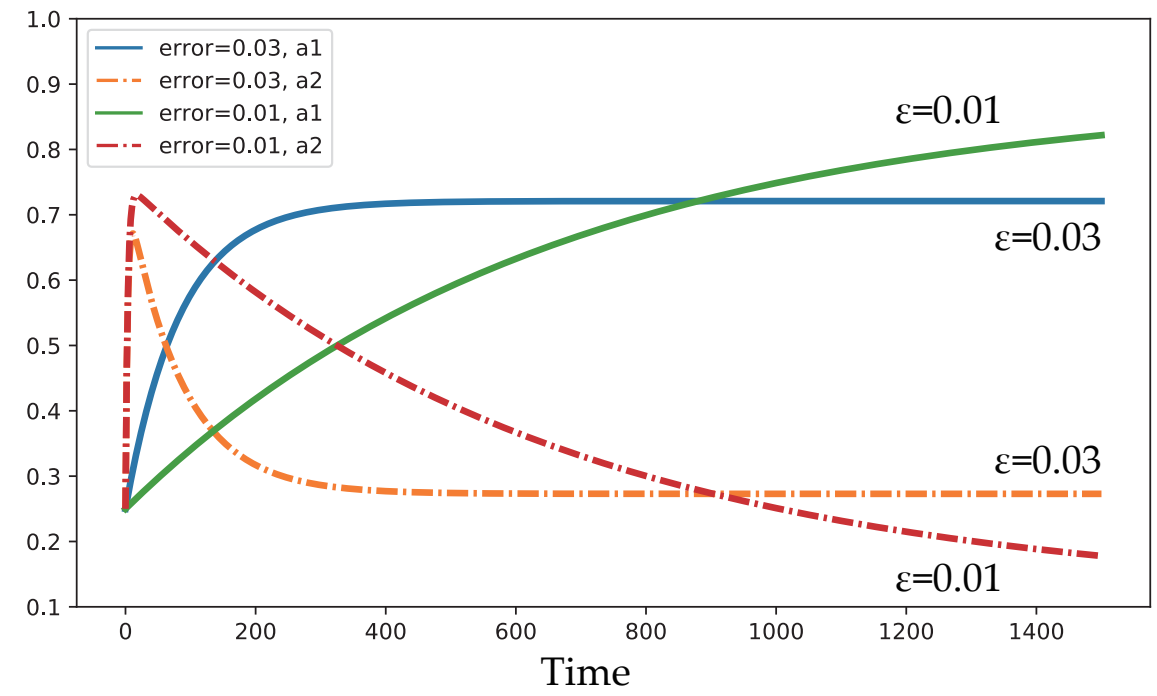

Figure 3: Probability of the Condorcet winner $a_{1}$ (solid line) and $a_{2}$ (dashed line)

quota increases. In particular, the core under the unanimity rule (the largest quota) coincides with the set of Pareto efficient policies. When the core includes multiple policies, the outcome of majority voting is indeterminate. This is sometimes called the indeterminacy problem (Caplin and Nalebuff 1988). Theorem 1 shows that the stochastic evolutionary theory resolves the indeterminacy problem in that the Condorcet winner is uniquely selected as a long-run equilibrium in a dynamic voting process.

Example 3. Recall the collective choice problem in Example 1, where 25 voters choose from 4 policies $\left\{a_{1}, \ldots, a_{4}\right\}$. Recall also that $\underline{q}=13$. Thereom 1 implies that the Condorcet winner $a_{1}$ is the unique long-run equilibrium under every $q$-majority rule with $q \geq 13$.

The intuitive reasoning behind the result for unanimity $(q=25)$ is as follows. Recall Figure 2 in Example 2, where the number associated with an arrow indicates the number of voters who oppose the transition. This number is actually the transition cost under unanimity (i.e., the number of mistakes required for the transition to occur). The likelihood of a transition decreases as the number of opposing voters increases. A long-run equilibrium policy must be the root of a tree that minimizes, among all trees, the sum of the opposing voters associated with its transitions.

The $a_{2}$-tree in Figure 2(a) minimizes the number of the opponents among all $a_{2}$-trees. It is, however, not a $\underline{q}$-majority tree as discussed in Example 2. The $a_{1}$-tree in Figure 2(b) is a $q$-majority tree that minimizes the number of opponents among all trees.

Figure 3 shows the dynamics of the probability of the Condorcet winner $a_{1}$ and another policy $a_{2}$ for $\varepsilon \in\{0.01,0.03\}$. The quota is $q=15$. All four policies are equiprobable in the initial distribution. The probability of $a_{1}$ increases over time for both error rates while the probability of 
$a_{2}$ first increases rapidly and then gradually decreases. It is more likely for the process to move to $a_{2}$ when the initial state is $a_{3}$ or $a_{4}$. It explains the initial increase of the probability of $a_{2}$. The probability of $a_{2}$, however, decreases afterwards because more voters prefer $a_{1}$ to $a_{2}-$ the transition from $a_{2}$ to $a_{1}$ is more likely than the opposite transition. Figure 3 illustrates that society eventually implements the Condorcet winner $a_{1}$ for both error rates. Note that the probability of $a_{1}$ will never reach one, owing to the error probability $\varepsilon>0$. As implied by Theorem 1 , the probability converges to one as $\varepsilon$ vanishes.

Theorem 1 assumes the Condorcet winner exists. We now consider a general case where the Condorcet winner may not exist. The set of long-run equilibria depends on a voting quota $q$. Let $\mathscr{A}_{\underline{q}}^{t c}$ be the top cycle under the $\underline{q}$-majority, and let $M_{q}\left(\mathscr{A}_{\underline{q}}^{t c}\right)$ be the set of stochastically stable policies when the policy space is restricted to $\mathscr{A}_{q}^{t c}$ and the voting rule is the $q$-majority.

Proposition 1. For all $q \geq \underline{q}$, a policy $z^{*}$ is stochastically stable under a $q$-majority if and only if $z^{*} \in M_{q}\left(\mathscr{A}_{\underline{q}}^{t c}\right)$.

According to this proposition, we can restrict the policy space to $\mathscr{A}_{\underline{q}}^{\text {tc }}$ to characterize the longrun equilibrium for every $q$-majority with $q \geq \underline{q}$. When $q=\underline{q}$, every policy in the top cycle $\mathscr{A}_{\underline{q}}^{t c}$ can be reached without error from any other policy. Thus, all policies in $\mathscr{A}_{\underline{q}}^{\text {tc }}$ are long-run equilibria, whereas those outside $\mathscr{A}_{\underline{q}}^{t c}$ are not. When $q>\underline{q}$, policies in $\mathscr{A}_{q}^{t c}$ may not be reached without error from policies outside $\mathscr{A}_{\underline{q}}^{t c}$. For example, some policies outside $\mathscr{A}_{\underline{q}}^{\text {tc }}$ may be in the core $C(q)$ when $q>\underline{q}$. Replacing such polices will incur positive transition costs. Nevertheless, we can ignore such policies to compute a stochastically stable policy, as shown in the following example.

Example 4. Consider a collective choice problem with 25 voters and four policies. The voters' preferences are summarized in Table 2. Observe that $\underline{q}=13$ and $\mathscr{A}_{\underline{q}}^{t c}=\left\{a_{1}, a_{2}, a_{3}\right\}$. Suppose

Table 2: Preference and voting matrices of 25 voters and four policies

\begin{tabular}{|c|c|}
\hline Preferences & \# of players \\
\hline$a_{1} \succ a_{4} \succ a_{2} \succ a_{3}$ & 8 \\
\hline$a_{2} \succ a_{3} \succ a_{4} \succ a_{1}$ & 6 \\
\hline$a_{3} \succ a_{1} \succ a_{2} \succ a_{4}$ & 11 \\
\hline
\end{tabular}

\begin{tabular}{|c|c|c|c|c|}
\hline & $a_{1}$ & $a_{2}$ & $a_{3}$ & $a_{4}$ \\
\hline$a_{1}$ & - & 19 & 8 & 19 \\
\hline$a_{2}$ & 6 & - & 14 & 17 \\
\hline$a_{3}$ & 17 & 11 & - & 17 \\
\hline$a_{4}$ & 6 & 8 & 8 & - \\
\hline
\end{tabular}

$q=20$, i.e. four-fifth majority votes. The cost of a transition is given by 20 minus the number of voters supporting the transition. The stochastically stable policy with the state space $\mathscr{A}_{\underline{q}}^{t c}$ is $a_{3}$. Proposition 1 implies that $a_{3}$ is the stochastically stable policy with the original state space $\mathscr{A}=\left\{a_{1}, \ldots, a_{4}\right\}$.

To see this, observe that $\left\{\left(a_{2}, a_{1}\right),\left(a_{3}, a_{2}\right),\left(a_{4}, a_{1}\right)\right\}$ is the $a_{1}$-tree with the minimum cost among all $a_{1}$-trees. Similarly, $\left\{\left(a_{1}, a_{3}\right),\left(a_{3}, a_{2}\right),\left(a_{4}, a_{1}\right)\right\}$ is the $a_{2}$-tree with the minimum cost 


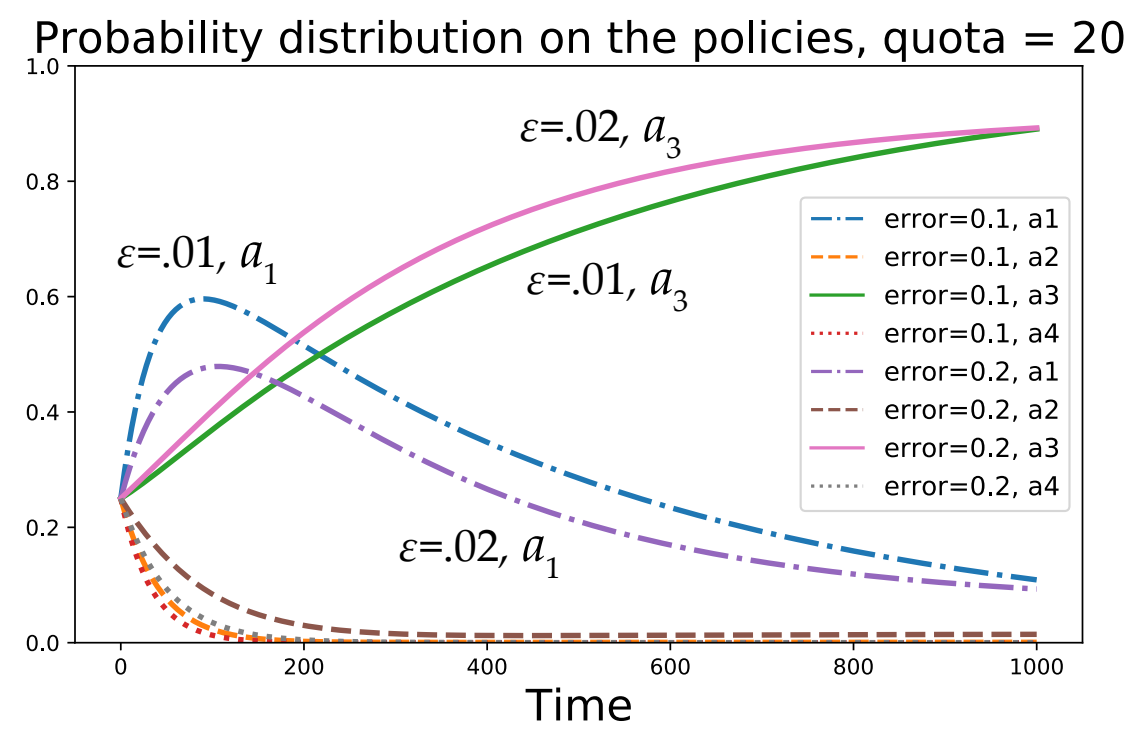

Figure 4: Probability of stochastically stable policy $a_{3}$ (solid line) and other policies (dashed line) for $q=20$

among all $a_{2}$-trees, and $\left\{\left(a_{1}, a_{3}\right),\left(a_{2}, a_{1}\right),\left(a_{4}, a_{1}\right)\right\}$ is the $a_{3}$-tree with the minimum cost among all $a_{3}$-trees. Because all trees include the same transition $\left(a_{4}, a_{1}\right)$, the difference in costs between these minimum-cost trees does not change, even if we remove policy $a_{4}$ from the state space. For example, the costs of the $a_{1}$-tree and the $a_{3}$-tree are 8 and 5 , respectively. If we exclude $a_{4}$, the costs change to 7 and 4, respectively, but the difference remains the same.

Figure 4 shows the dynamics of the probability of the four policies for $q=20$ and $\varepsilon \in$ $\{0.1,0.2\} . a_{3}$ (solid line) is uniquely stochastically stable for $q=20$. All four policies are equiprobable in the initial distribution. The probability of $a_{1}$ has a rapid increase in initial periods. This is due to that the transitions from $a_{2}$ and $a_{4}$ to $a_{1}$ are the most likely. The probability of $a_{1}$, however, keeps decreasing after that, and the probability eventually accumulates on the stochastically stable policy $a_{3}$ for both error probabilities.

Proposition 1 has two implications. First, it shows the robustness of the long-run equilibria. The top cycle $\mathscr{A}_{\underline{q}}^{t c}$ does not change, even if we add policies to $\mathscr{A}$ that are defeated by every $a \in$ $\mathscr{A}_{\underline{q}}^{t c}$. Second, it simplifies the computation of a long-run equilibrium. In general, it is bothersome but necessary to consider transition costs for all policies outside $\mathscr{A}_{\underline{q}}^{t c}$. However, this proposition implies that it is sufficient for us to compute transitions between policies in $\mathscr{A}_{\underline{q}}^{t c}$. 


\section{Multidimensional Policy Space}

We assume a collective choice problem where policies are in the $h$-dimensional Euclidean space $\mathbb{R}^{h}$. With this additional assumption, we can further characterize stochastically stable policies beyond Proposition 1. For a policy $a \in \mathbb{R}^{h}$ and $i=1, \cdots, h$, the $i$-th coordinate of $a$ represents its position on the $i$-th issue. Every voter has a Euclidean preference over policies; each player $i \in N$ has an ideal point, denoted as $s_{i} \in \mathbb{R}^{h}$, and he/she prefers policies closer to the ideal point. Formally, player $i$ 's utility function $u_{i}$ satisfies $u_{i}(a)>u_{i}\left(a^{\prime}\right)$ if and only if $d\left(a, s_{i}\right)<d\left(a^{\prime}, s_{i}\right)$, where $d(a, b)$ denotes the (Euclidean) distance between $a$ and $b$. The Euclidean preference generalizes the "single-peaked" preference of Black (1948). Here, $\bar{n}$ and $\mathfrak{C}^{*}$ denote the min-max quota and the min-max set, respectively, in $\mathbb{R}^{h}$. That is, $\bar{n}=\min _{r \in \mathbb{R}^{h}} \max _{r^{\prime} \in \mathbb{R}^{h}} n\left(r, r^{\prime}\right)$ and $\mathfrak{C}^{*}=\left\{r \in \mathbb{R}^{h}\right.$ : $\left.\max _{r^{\prime} \in \mathbb{R}^{h}} n\left(r, r^{\prime}\right)=\bar{n}\right\}$.

To apply our stochastic evolutionary theory, we approximate $\mathbb{R}^{h}$ by a finite subset of itself. Let $\mathscr{A}^{0} \subset \mathbb{R}^{h}$ be a bounded convex set. Let $\mathscr{A}^{\delta} \subset \mathscr{A}^{0}$ be a finite approximation of $\mathscr{A}^{0}$, with maximum distance $\delta>0$; that is, for every $r \in \mathscr{A}^{0}$, there exists some $a \in \mathscr{A}^{\delta}$ such that $d(a, r)<\delta$. We consider a long-run equilibrium when the finite space $\mathscr{A}^{\delta}$ approximates $\mathbb{R}^{h}$ well. Specifically, we characterize stochastically stable policies over $\mathscr{A}^{\delta}$ when $\mathscr{A}^{0}$ is sufficiently large and the approximation $\delta$ converges to zero.

In our analysis, we assume that (i) $s_{i} \in \mathscr{A}^{0}$, for all $i \in N$; and (ii) $\mathscr{A}^{\delta} \cap \mathfrak{C}^{*} \neq \emptyset$. The first assumption ensures that the min-max set $\mathfrak{C}^{*}$ defined over $\mathbb{R}^{h}$ is included in $\mathscr{A}^{0}$. The second assumption means that the approximation is sufficiently fine that some point of the minmax set $\mathfrak{C}^{*}$ is included in $\mathscr{A}^{\delta}$. For $\mathscr{A}^{\delta}$, we define the min-max quota and the min-max set as $\bar{n}^{\delta}=\min _{a \in \mathscr{A} \delta} \max _{a^{\prime} \in \mathscr{A} \delta} n\left(a, a^{\prime}\right)$ and $\mathscr{A}^{m m, \delta}=\left\{a \in \mathscr{A}^{\delta}: \max _{a^{\prime} \in \mathscr{A}^{\delta}} n\left(a, a^{\prime}\right)=\bar{n}^{\delta}\right\}$, respectively.

Lemma B.4 in the Appendix shows that any pair of policies can be connected via a sequence of pairwise voting under a $\underline{q}$-majority if $\mathscr{A}^{0}$ is sufficiently large and $\delta$ is sufficiently small. This suggests that the top cycle $\mathscr{A}_{\underline{q}}^{\text {tc }}$ can be arbitrarily large, and that Proposition 1 barely helps characterize stochastically stable policies. Nevertheless, the next theorem characterizes the set of stochastically stable policies for all $q$-majority rules, where $q \in\{\underline{q}, \cdots, n\}$.

Theorem 2. Fix any $\rho>0$, and let $B^{\delta}(\rho)=\left\{a \in \mathscr{A}^{\delta}:\|a\|<\rho\right\}$.

(i) For $q \leq \bar{n}$, every $a \in B^{\delta}(\rho)$ is stochastically stable for every sufficiently small $\delta$ and every sufficiently large $\mathscr{A}^{0}$.

(ii) For $q>\bar{n}, \lim _{\delta \rightarrow 0} \lim _{\mathcal{\varepsilon} \rightarrow 0} \pi_{\varepsilon}^{q}\left(\mathscr{A}^{m m, \delta}\right)=1$ for every sufficiently large $\mathscr{A}^{0}$.

The theorem shows the following properties of the long-run equilibrium when the policy space is multidimensional. The set of stochastically stable policies differs, depending on whether $q \leq \bar{n}$. When $q \leq \bar{n}$, every policy in an arbitrarily large open ball $B^{\delta}(\rho)$ is stochastically stable, provided that an approximation of the policy space is sufficiently large and fine. Intuitively, every policy 
inside such an open ball is in the top cycle under a $q$-majority, and thus can be connected via zero-cost transitions. When $q>\bar{n}$, the set of stochastically stable policies is approximately equal to the min-max set $\mathscr{A}^{m m, \delta}$. The result generalizes Theorem 1 to the context of multidimensional policy spaces. If the Condorcet winner $a^{C W}$ exists, then $\bar{n}<n / 2$. Thus, $\mathscr{A}^{m m, \delta}=\left\{a^{C W}\right\}$, for every $q \geq n / 2$ and every $\delta$.

Theorem 2 has the following implications for the two issues of intransitivity and indeterminacy related to majority voting. In general, there is a trade-off between intransitivity and indeterminacy. When $q \leq \bar{n}$, the social preference order under the $q$-majority rule violates transitivity (McKelvey, 1976). As a result, intransitivity occurs; the $q$-majority core does not exist and all policies are in the top cycle under $q$-majority. When $q>\bar{n}$, the intransitivity of majority voting is mitigated, because the $q$-majority core includes at least the min-max policies. However, as the quota becomes larger, we face indeterminacy, because the core may include many policies. In fact, the $q$-majority core is, in general, larger than the min-max set for all $q>\bar{n}+1$. To overcome both issues, we should skillfully choose $q=\bar{n}+1$, such that the core exists and its size is a minimum; in this case, the core coincides with the min-max set $\mathscr{A}^{m m, \delta}$. Caplin and Nalebuff (1988) show that it is safe to choose $q=.64 n$ to avoid intransitivity. The problem is that $\bar{n} / n \approx .64$ is the worst case, and the min-max quota $\bar{n}$ may be much lower than $64 \%$ of $n$. As a result, many consider that, in such cases, choosing $q=.64 n$ will aggravate indeterminacy. Theorem 2 (ii) implies that stochastic evolutionary theory resolves the indeterminacy of majority voting in that only policies in the min-max set can be longrun equilibria for all $q \geq \bar{n}+1$.

The next corollary follows immediately when Theorem 2 is applied to the unidimensional case. The result corresponds to the mean voter's theorem of Black (1948).

Corollary 1. Let $s^{*}$ be the mean voter's ideal point in a unidimensional problem $(h=1)$, and let $n$ be an odd number. Then, $\lim _{\delta \rightarrow 0} \lim _{\varepsilon \rightarrow 0} \pi_{\varepsilon}^{q}\left(s^{*}\right)=1$, for every $q \geq q$ and every sufficiently large $\mathscr{A}^{0}$.

Finally, we give an example that shows the min-max set for a multidimensional policy space.

Example 5 (min-max sets). Figure 5(a) shows the min-max set (the gray area) for a collective choice problem with three players and a policy space on $\mathbb{R}^{2}$. The players' ideals points are depicted by $s_{1}, s_{2}$, and $s_{3}$. Note that the min-max quota is $\bar{n}=2$. The min-max set coincides with the convex hull of the ideal points. For any policy outside the min-max set, there is a policy that $\bar{n}+1$ players strictly prefer. For example, three players strictly prefer $a_{2}$ to $a_{1}$ in Figure 5(a). By contrast, at least $n-\bar{n}$ players vote against any move from a policy within the min-max set. For example, the player with $s_{1}$ votes against the move from $a_{3}$ to $a_{4}$.

Figures 5(b) and 5(c) show the min-max sets for settings with four and five players, respectively. Note that $\bar{n}=2$ for the four-player setting, and $\bar{n}=3$ for the five-player setting. These 


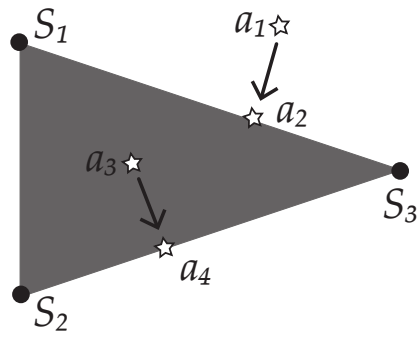

(a) 3 players

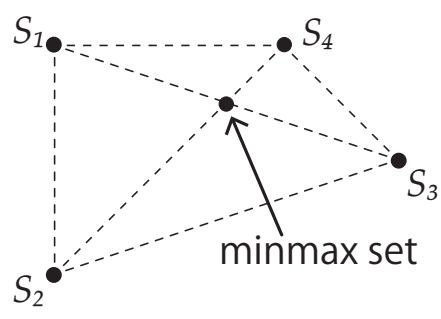

(b) 4 players

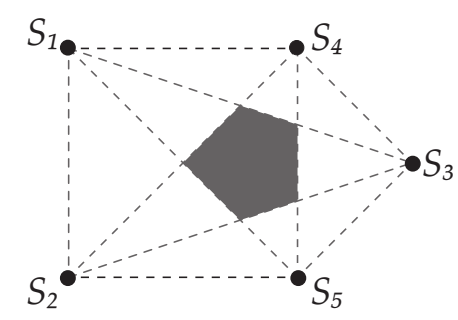

(c) 5 players

Figure 5: The min-max sets

min-max sets are the intersection of the convex hulls of all $\bar{n}+1$ players' ideal points. In the fourplayer setting, the min-max set is the intersection of the diagonals. This point is not a Condorcet winner, but is undefeated under the simple majority $q=3$. Our result shows that a dynamic process of majority voting with $q \geq 3$ will choose this unique undefeated policy in the long run.

\section{Logit Choice and Borda Winner}

Thus far, we have characterized stochastically stable policies of the BRM. However, because the behavior of boundedly rational players is diverse, and cannot be described by a unique choice model, it is important that we examine how the choice rule affects the set of stochastically stable policies. Here, we examine the well-documented logit choice as an alternative choice rule for boundedly rational individuals.

Following Blume (1993), suppose that players employ the logit choice rule with noise level $\eta>0$. Given the status quo $a$ and a proposal $a^{\prime}$, the choice probability of voting for $a^{\prime}$ is given by

$$
\Psi_{j}^{\eta}\left(a, a^{\prime}\right)=\frac{\exp \left(\eta^{-1} u_{j}\left(a^{\prime}\right)\right)}{\exp \left(\eta^{-1} u_{j}\left(a^{\prime}\right)\right)+\exp \left(\eta^{-1} u_{j}(a)\right)} .
$$

The logit choice rule can be derived from a random utility model in which the utility for each policy is perturbed by i.i.d. random variables with the Gumbel distribution. The distribution is bell-shaped, and is similar to a normal distribution. This choice rule is amenable to computation, and approximates a random utility model with normally distributed noise.

For two policies $a$ and $a^{\prime}$, the transition probability from $a$ to $a^{\prime}$ under the logit choice rule is given by replacing the BRM choice rule $\Psi_{j}^{\varepsilon}$ with the logit function $\Psi_{j}^{\eta}$. Let $P_{a, a^{\prime}}^{\eta, q}$ denote the transition probability with $\Psi_{j}^{\eta}\left(a, a^{\prime}\right)$, and let $\pi_{\eta}^{q}$ be the stationary distribution under $P_{.,}^{\eta, q}$. We define the stochastic stability for the logit choice rule in the same manner as that for the BRM. A policy $z$ is stochastically stable under a q-majority with the logit choice if $\lim _{\eta \rightarrow 0} \pi_{\eta}^{q}(z)>0$. 
For the logit choice, it is known that the unlikeliness of a player $i$ 's choice is given by ${ }^{14}$

$$
c_{i}\left(a, a^{\prime}\right)=-\lim _{\eta \rightarrow 0} \eta \log \Psi_{i}^{\eta}\left(a, a^{\prime}\right)=\max \left\{u_{i}(a)-u_{i}\left(a^{\prime}\right), 0\right\}
$$

The transition cost from $a$ to $a^{\prime}$ under a $q$-majority is given as

$$
c_{a a^{\prime}}^{q}=\min _{J \in N_{q}} \sum_{i \in J} \max \left\{u_{i}(a)-u_{i}\left(a^{\prime}\right), 0\right\}
$$

where $N_{q}$ denotes the set of subsets of $N$ with size $q$. The cost $c_{a a^{\prime}}^{q}$ represents the unlikeliness of the transition $\left(a, a^{\prime}\right)$. That is, the unlikeliness is the smallest sum of the payoff deficits for $q$ players whose acceptance is needed for proposal $a^{\prime}$ to win.

Stochastic stability under the logit choice favors the utilitarian social welfare function. The next theorem shows that every stochastically stable policy of the logit choice rule under the unanimity rule maximizes the social welfare function $\mathscr{P}(a)$ (i.e., the sum of all players' utilities). ${ }^{15}$

Theorem 3. Let $\mathscr{P}(a) \equiv \sum_{i \in N} u_{i}(a)$, for all $a \in \mathscr{A}$. A policy is stochastically stable under the unanimity rule with the logit choice if and only if it maximizes $\mathscr{P}(\cdot)$ among all policies.

We introduce the Borda winner, which is based on a voting scoring method. Each player ranks the policies in order of his/her preferences. The rankings are converted into points; a policy receives one point for being ranked last, two for being next-to-last, and so on, up to $|\mathscr{A}|$ points for being ranked first. The Borda winner is the policy with the highest total score.

A maximizer of the social welfare function may correspond to the winner in some voting scoring methods, depending on the players' preferences. Specifically, when the players' utility scores for policies are linearly increasing with their rankings, a policy is a long-run equilibrium if and only if it is a Borda winner.

Corollary 2. Suppose that every player has linear preferences over all policies, in increments of one, according to his preference order; that is, $u_{i}(a) \in\{1,2, \ldots,|\mathscr{A}|\}$, for all $i \in N$ and $a \in \mathscr{A}$. The set of stochastically stable policies of the logit choice under the unanimity rule coincides with the set of Borda winners.

This contrasts with the result of Theorem 1, which shows the stochastic stability of the Condorcet winner under the BRM for all majority rules. The Borda winner can emerge as a long-run equilibrium if the logit choice better captures the behavior of boundedly rational voters. This result provides a dynamic foundation for the Borda winner. Suppose a society in which policies are

\footnotetext{
${ }^{14}$ See Alós-Ferrer and Netzer (2010), for example. See also Sawa (2014) for the unlikeliness of joint deviations.

${ }^{15}$ The result resembles that of Kandori et al. (2008), who study exchange economies. They show that an allocation that maximizes the sum of utility functions is stochastically stable under the logit choice.
} 
formed under the unanimity rule; that is, everyone has an equal right of veto. Here, the Borda winner would be selected in the long run in this hypothetical society, provided that those with linear preferences follow the logit choice. The Borda method can be viewed as a mechanism for implementing such a long-run equilibrium.

\section{Conclusion}

We have considered a dynamic policy-making process, where a status quo policy is repeatedly challenged by an opposing policy. Our analysis is based on stochastic evolutionary game theory. We have shown that the Condorcet winner is a unique long-run equilibrium for all majority rules. When the policy space is multidimensional, a long-run equilibrium under (super-)majority voting must belong to the min-max set if the voting quota $q$ is larger than the min-max quota. The stochastic evolutionary game theory provides new insight into the theory of social choice. Specifically, the theory mitigates the indeterminacy problem of majority voting.

To conclude the paper, we comment on the limitations of our research, and offer possible directions for future work. A voting rule is given exogenously in our model. Thus, it would be interesting to study an endogenous choice of voting rules from an evolutionary perspective. ${ }^{16}$ Voters are myopic and their choices do not include strategic considerations. Although these assumptions are common in the literature on evolutionary game theory, they are restrictive. A more elaborate model of boundedly rational voting should be developed in collaboration with behavioral and experimental economics. It may be worthwhile combining our model with empirical behavioral error models, such as those of Lim and Neary (2016), Mäs and Nax (2016), and Hwang et al. (2018). Finally, a further comparative study of normative and evolutionary approaches to the theory of social choice is worth studying.

\section{Appendix A}

\section{Transition Probabilities in the Dynamic Process}

Recall that Eq. (1) is the players' choice rule when they make no error, and Eq. (2) is their rule when their choices are stochastic. Let $P_{a, a^{\prime}}^{0, q}$ denote the transition probability from $a$ to $a^{\prime}$ under a $q$-majority when the choice rule is Eq. (1). Let $p_{a, a^{\prime}}$ be the probability that a proposal $a^{\prime} \in \mathscr{A}$ is

\footnotetext{
${ }^{16} \mathrm{~A}$ few studies have examined endogenous voting rules (see Barbera and Jackson (2004) and Aghion et al. (2004)).
} 
made against the status quo policy $a$. Then, $P_{a, a^{\prime}}^{0, q}$ can be expressed as follows:

$$
P_{a, a^{\prime}}^{0, q}=\left\{\begin{array}{ll}
p_{a, a^{\prime}} & \text { if } n\left(a, a^{\prime}\right) \geq q, \\
0 & \text { otherwise. }
\end{array} \quad \forall a, a^{\prime} \in \mathscr{A} \text { with } a \neq a^{\prime}\right.
$$

The probability of remaining with the status quo $a$ is $P_{a, a}^{0, q}=1-\sum_{a^{\prime} \neq a} P_{a, a^{\prime}}^{0, q}$. We call the Markov chain $P^{0, q}=\left(P_{a, a^{\prime}}^{0, q}\right)$ an unperturbed process under a $q$-majority.

Let $\theta \subseteq \mathscr{A}$. Here, $\theta$ is a recurrent class of the unperturbed process $P^{0, q}$ under a $q$-majority if it satisfies the following conditions:

1. For all $a_{1}, a_{k} \in \theta$, there exists a sequence of policies $\left\{a_{1}, \ldots, a_{k}\right\} \subseteq \theta$, such that $P_{a_{i}, a_{i+1}}^{0, q}>0$, for all $i \in\{1, \ldots, k-1\}$.

2. For all $a \in \theta$ and $a^{\prime} \in \mathscr{A} \backslash \theta, P_{a, a^{\prime}}^{0, q}=0$.

As mentioned in the text, the set of recurrent classes coincides with the myopic stable set $M S S_{q}$ (Theorem 3.16 of Demuynck et al. 2019). We have the following result for top cycles (the proof is omitted).

Lemma A.1 (Top cycle and recurrent class).

(i) A top cycle under a q-majority is a unique recurrent class of the unperturbed process $P^{0, q}$.

(ii) For every $q \leq \underline{q}$, the unperturbed process $P^{0, q}$ has a unique recurrent class.

Let $P_{a, a^{\prime}}^{\varepsilon, q}$ denote the transition probability from $a$ to $a^{\prime}$ under a $q$-majority when the choice rule is Eq. (2). We call the Markov chain $P^{\varepsilon, q}=\left(P_{a, a^{\prime}}^{\varepsilon, q}\right)$ a perturbed process under a $q$-majority. Let $m$ be the number of voters who prefer a policy $a^{\prime}$ to the status quo $a$. Then, the transition probabilities are expressed as follows:

$$
P_{a, a^{\prime}}^{\varepsilon, q}=p_{a, a^{\prime}} \sum_{k=q}^{n} \sum_{j=0}^{m} C(m, j) C(n-m, k-j)(1-\varepsilon)^{j} \varepsilon^{m-j} \varepsilon^{k-j}(1-\varepsilon)^{n-m-(k-j)},
$$

where $C(K, k)$ denotes the number of $k$-combinations from $K$ elements. We use the convention that $C(K, k)=0$ if $k<0$. Because every player votes for a proposal with positive probability, we need to sum the probabilities of all events in which at least $q$ players vote for the proposal. Rather than considering the exact probabilities, we focus on the highest power of $\varepsilon$ for each probability.

\section{The Computation Method}

The key computation method for long-run equilibria was developed as part of stochastic evolutionary game theory (Foster and Young, 1990; Kandori et al., 1993; Young, 1993). We first define 
the notion of a transition cost from one policy to another, which measures the unlikeliness of the transition occurring. The transition cost from $a$ to $a^{\prime}$ under a $q$-majority is defined as

$$
c_{a a^{\prime}}^{q}=\max \left\{q-n\left(a, a^{\prime}\right), 0\right\}
$$

Recall that $n\left(a, a^{\prime}\right)$ is the number of players who prefer $a^{\prime}$ to $a$. The transition cost $c_{a a^{\prime}}^{q}$ from policy $a$ to $a^{\prime}$ under a $q$-majority is the minimum number of mistakes ("mutations," in evolutionary terminology) required for at least $q$ players to vote for $a^{\prime}$. Some of these players may not prefer $a^{\prime}$ to the status quo $a$, but they may accept proposal $a^{\prime}$ "by mistake." If this is the case, then the transition $\operatorname{cost} c_{a a^{\prime}}^{q}$ will be positive, and equal to the minimum necessary number of mistakes. If $q$ players who prefer $a^{\prime}$ to $a$ exist, then $c_{a a^{\prime}}^{q}$ is zero.

For two policies $a$ and $a^{\prime}$, we denote the transition from $a$ to $a^{\prime}$ as notation $\left(a, a^{\prime}\right)$. We call a set of transitions $\left\{\left(a_{1}, a_{2}\right),\left(a_{2}, a_{3}\right), \ldots,\left(a_{L-1}, a_{L}\right)\right\}$ a path from $a_{1}$ to $a_{L}$ on $\mathscr{A}$ if $a_{i} \neq a_{j}$, for all $i \neq j$. Note that any transition $\left(a_{i}, a_{i+1}\right)$ may occur with a positive probability under a perturbed process. For $a \in \mathscr{A}$, we call a set of transitions, denoted as $\tau_{a}$, an $a$-tree if there exists a unique path from $a^{\prime}$ to $a$ for all $a^{\prime} \in \mathscr{A}$, with $a^{\prime} \neq a$. Let $\Upsilon_{a}$ denote the set of all $a$-trees. Given an $a$-tree $\tau_{a}$ and a majority quota $q$, we define the cost of the $a$-tree $\tau_{a}$ as

$$
c_{q}\left(\tau_{a}\right)=\sum_{(v, w) \in \tau_{a}} c_{v w}^{q}
$$

We define $c_{q}^{*}(a)$ as the lowest cost from among all $a$-trees, and define $m_{q}^{*}$ as the minimum of $c_{q}^{*}(a)$ from among all policies $a$ in $\mathscr{A}$. That is,

$$
c_{q}^{*}(a)=\min _{\tau_{a} \in \Upsilon_{a}} c_{q}\left(\tau_{a}\right), \quad m_{q}^{*}=\min _{a \in \mathscr{A}} c_{q}^{*}(a) .
$$

Define

$$
M_{q}=\left\{a \in \mathscr{A}: c_{q}^{*}(a)=m_{q}^{*}\right\}
$$

The following theorem is proved by Kandori et al. (1993) and Young (1993) in our context.

Theorem A.1. A policy $a \in \mathscr{A}$ is stochastically stable if and only if $a \in M_{q}$.

The theorem states that a policy is stochastically stable if and only if a tree with itself as the root has the minimum cost among all trees. An implication of this is that a stochastically stable policy $a \in \mathscr{A}$ under a $q$-majority must belong to a recurrent class of the unperturbed process. If $a$ is not in any recurrent class, then there exists a path from $a$ to some recurrent class with zero cost. The cost of any $a$-tree must be greater than that of a $b$-tree, for every policy $b$ in the recurrent class. 


\section{Appendix B}

\section{Proofs of Analytic Results}

Recall that a tree $\tau_{a}$ for $a \in \mathscr{A}$ is $\underline{q}$-majority tree if $n\left(a^{\prime}, a^{\prime \prime}\right) \geq \underline{q}$, for all $\left(a^{\prime}, a^{\prime \prime}\right) \in \tau_{a}$.

Proof of Lemma 1. Fix $q \geq \underline{q}$. Assume that $a \in \mathscr{A}$ is stochastically stable under a $q$-majority. By way of contradiction, suppose that $a$ has no $q$-majority $a$-tree. Then, for every $a$-tree $\tau_{a}$, there exists some transition $\left(a^{\prime}, a^{\prime \prime}\right) \in \tau_{a}$, such that $n\left(a^{\prime}, a^{\prime \prime}\right)<\underline{q}$. If $n\left(a^{\prime}, a\right) \geq \underline{q}$, for all such transitions $\left(a^{\prime}, a^{\prime \prime}\right)$ in $\tau_{a}$. Then, one can construct a $\underline{q}$-majority $a$-tree by replacing all such $\left(a^{\prime}, a^{\prime \prime}\right)$ with $\left(a^{\prime}, a\right)$. This contradicts the supposition. If there exists some transition $\left(a^{\prime}, a^{\prime \prime}\right) \in \tau_{a}$ such that $n\left(a^{\prime}, a^{\prime \prime}\right)<\underline{q}$ and $n\left(a^{\prime}, a\right)<\underline{q}$, then remove $\left(a^{\prime}, a^{\prime \prime}\right)$ from $\tau_{a}$, and add $\left(a, a^{\prime}\right)$. The resulting set of edges must be an $a^{\prime}$-tree, denoted as $\tau_{a^{\prime}}$. From (7), its cost is given by

$$
c_{q}\left(\tau_{a^{\prime}}\right)=c_{q}\left(\tau_{a}\right)-\left(q-n\left(a^{\prime}, a^{\prime \prime}\right)\right)+\max \left\{q-n\left(a, a^{\prime}\right), 0\right\}
$$

Note that $q-n\left(a^{\prime}, a^{\prime \prime}\right)>0$, because $q \geq \underline{q}>n\left(a^{\prime}, a^{\prime \prime}\right)$. Observe that

$$
q-n\left(a^{\prime}, a^{\prime \prime}\right)>q-\underline{q} \geq q-n\left(a, a^{\prime}\right)
$$

The final inequality holds because $n\left(a^{\prime}, a\right)<\underline{q}$ implies $n\left(a, a^{\prime}\right) \geq \underline{q}$. Then, we have $c_{q}\left(\tau_{a^{\prime}}\right)<c_{q}\left(\tau_{a}\right)$. This contradicts that $a$ is stochastically stable under the $q$-majority.

Proof of Theorem 1. Suppose that a Condorcet winner $a^{C W}$ exists. It is easy to see that any $a \neq a^{C W}$ has no $q$-majority $a$-tree. Any $a$-tree must include a transition from $a^{C W}$, say $\left(a^{C W}, a^{\prime}\right)$. Because $n\left(a^{C W}, a^{\prime}\right)<\underline{q}$, any $a \neq a^{C W}$ has no $\underline{q}$-majority $a$-tree. Then, Lemma 1 implies the claim.

We prove Proposition 1. Lemma A.1 shows that if a quota $q$ is not greater than $q$, then the unperturbed process has a unique recurrent class that coincides with the top cycle. This proves the claim of Proposition 1 for $q=\underline{q}$. Thus, we focus on the claim of Proposition 1 for $q>\underline{q}$. Recall that $\mathscr{A}_{\underline{q}}^{t c}$ is the top cycle under a $\underline{q}$-majority. Let $\Upsilon_{a}\left(\mathscr{A}_{\underline{q}}^{t c}\right)$ denote the set of $a$-trees over $\mathscr{A}_{\underline{q}}^{t c}$, and define

$$
\begin{aligned}
& c_{q, \mathscr{A}_{\underline{q}}^{t c}}^{*}(a)=\min _{\tau_{a} \in \Upsilon_{a}\left(\mathscr{A}_{\underline{q}}^{t c}\right)} c_{q}\left(\tau_{a}\right), \quad c_{q, \mathscr{A}_{\underline{q}}^{t c}}^{*}=\min _{a \in \mathscr{A}_{\underline{\underline{q}}}^{t c}} c_{q, \mathscr{A}_{\underline{\underline{q}}}^{t c}}^{*}(a), \\
& M_{q}\left(\mathscr{A}_{\underline{q}}^{t c}\right)=\left\{a \in \mathscr{A}_{\underline{q}}^{t c}: c_{q, \mathscr{A}_{\underline{q}}^{t c}}^{*}(a)=c_{q, \mathscr{A}_{\underline{q}}^{t c}}^{*}\right\} .
\end{aligned}
$$

We first prove Lemmas B.1 and B.2. The former shows that if a policy $a$ is stochastically stable for quota $q>\underline{q}, a$ must be in the top cycle under the $\underline{q}$-majority; that is, $a \in \mathscr{A}_{\underline{q}}^{t c}$. The latter shows 
that any minimum cost tree of stochastically stable policies has no edge emanating from some policy in $\mathscr{A}_{\underline{q}}^{t c}$ to one not in $\mathscr{A}_{\underline{q}}^{t c}$.

Lemma B.1. $\lim _{\varepsilon \rightarrow 0} \pi_{\varepsilon}^{q}(a)>0$ for $q>\underline{q}$ only if $a \in \mathscr{A}_{\underline{q}}^{t c}$.

Proof. By way of contradiction, suppose that $\pi_{\varepsilon}^{q}(a)>0$ for $a \notin \mathscr{A}_{q}^{t c}$. Note that $n\left(a, a^{\prime}\right)=n-$ $n\left(a^{\prime}, a\right)>n-\underline{q}$, for all $a^{\prime} \in \mathscr{A}_{\underline{q}}^{t c}$, because $a \notin \mathscr{A}_{\underline{q}}^{t c}$ implies that $n\left(a^{\prime}, a\right)<\underline{q}$, for $a^{\prime} \in \mathscr{A}_{\underline{q}}^{t c}$.

Let $\tau_{a}$ be an $a$-tree minimizing its cost; that is, $c_{q}\left(\tau_{a}\right)=m_{q}^{*}$. Choose $\left(a^{\prime}, a^{\prime \prime}\right) \in \tau_{a}$ such that $a^{\prime} \in \mathscr{A}_{\underline{q}}^{t c}$ and $a^{\prime \prime} \notin \mathscr{A}_{\underline{q}}^{t c}$. Such an edge must exist because the root of $\tau_{a}$ is not in $\mathscr{A}_{\underline{q}}^{t c}$. Remove $\left(a^{\prime}, a^{\prime \prime}\right)$ from $\tau_{a}$. This will reduce the cost of $\tau_{a}$ by at least $q-(\underline{q}-1)$, because $n\left(a^{\prime}, a^{\prime \prime}\right)<\underline{q}$ for $a^{\prime} \in \mathscr{A}_{\underline{q}}^{t c}$ and $a^{\prime \prime} \notin \mathscr{A}_{\underline{q}}^{t c}$. Then, add an edge $\left(a, a^{\prime}\right)$ to $\tau_{a}$. This will increase the cost by $q-n\left(a, a^{\prime}\right)$, which is at most $q-\underline{q}$. The resulting tree is an $a^{\prime}$-tree with a cost strictly less than $c_{q}\left(\tau_{a}\right)$. This contradicts that $c_{q}\left(\tau_{a}\right)=m_{q}^{*}$.

Lemma B.2. Suppose that $a \in \mathscr{A}$ is stochastically stable with $q>\underline{q}$. Let $\tau_{a}$ be such that $c_{q}\left(\tau_{a}\right)=$ $m_{q}^{*}$. If $a^{\prime} \in \mathscr{A}_{\underline{q}}^{t c}$ and $\left(a^{\prime}, a^{\prime \prime}\right) \in \tau_{a}$, then $a^{\prime \prime} \in \mathscr{A}_{\underline{q}}^{t c}$.

Proof. Observe that Lemma B.1 implies that $a \in \mathscr{A}_{q}^{t c}$. By way of contradiction, suppose there exists $\left(a^{\prime}, a^{\prime \prime}\right) \in \tau_{a}$, such that $a^{\prime} \in \mathscr{A}_{\underline{q}}^{t c}$ and $a^{\prime \prime} \notin \mathscr{A}_{\underline{q}}^{t c}$. Because $a^{\prime \prime} \notin \mathscr{A}_{\underline{q}}^{t c}, n\left(a^{\prime}, a^{\prime \prime}\right)<\underline{q}$. Remove edge $\left(a^{\prime}, a^{\prime \prime}\right)$ from $\tau_{a}$. This will reduce the cost of $\tau_{a}$ by at least $q-q+1$. Let $\tau_{a}^{1}$ denote the resulting set of edges.

If $n\left(a^{\prime}, a\right) \geq \underline{q}$, add edge $\left(a^{\prime}, a\right)$ to $\tau_{a}^{1}$. This will increase the cost by at most $q-\underline{q}$. The resulting set is an $a$-tree, say $\tau_{a}^{2}$. Observe that $c_{q}\left(\tau_{a}^{2}\right) \leq c_{q}\left(\tau_{a}\right)-(q-\underline{q}+1)+q-\underline{q}=c_{q}\left(\tau_{a}\right)-1$.

If $n\left(a^{\prime}, a\right)<q$, it must be that $n\left(a, a^{\prime}\right) \geq q$. Add edge $\left(a, a^{\prime}\right)$ to $\tau_{a}^{1}$. This will increase the cost by at most $q-q$. The resulting set is an $a^{\prime}$-tree, say $\tau_{a^{\prime}}$. Observe that $c_{q}\left(\tau_{a^{\prime}}\right) \leq c_{q}\left(\tau_{a}\right)-(q-\underline{q}+$ 1) $+q-\underline{q}=c_{q}\left(\tau_{a}\right)-1$. These observations contradict that $c_{q}\left(\tau_{a}\right)=m_{q}^{*}$.

Now, we are ready to prove Proposition 1.

Proof of Proposition 1. Let $\tau_{a}$ be an $a$-tree over $\mathscr{A}$. We say that $\tau_{a}$ has an $a$-subtree over a subset $\mathscr{A}^{\prime} \subset \mathscr{A}$ if, for all $a_{1} \in \mathscr{A}^{\prime}$, there exists a path $\left\{a_{1}, \ldots, a_{k}\right\} \subseteq \tau_{a}$, such that $a_{k}=a$ and $a_{i} \in \mathscr{A}^{\prime}$, for all $i \in\{1, \ldots, k\}$. Lemma B.1 implies that $a \notin \mathscr{A}_{\underline{q}}^{t c}$ cannot be stochastically stable. Lemma B.2 implies that if $a$ is stochastically stable, for $q>\underline{q}$, then $\tau_{a}$ minimizing $c_{q}(\cdot)$ must have an $a$-subtree over $\mathscr{A}_{q}^{t c}$. The proof for each part is conducted by way of contradiction.

"only if" part: Suppose that $a \in \mathscr{A}_{\underline{q}}^{t c} \backslash M_{q}\left(\mathscr{A}_{\underline{q}}^{t c}\right)$ is stochastically stable. Let $\tau_{a}$ be the minimum cost tree for $a$. Lemma B.2 implies that $\tau_{a}$ has an $a$-subtree over $\mathscr{A}_{q}^{t c}$, say $\tau_{a}^{\sharp}$.

Let $b \in M_{q}\left(\mathscr{A}_{\underline{q}}^{t c}\right)$, with $\tau_{b}^{\sharp}$ a $b$-subtree over $\mathscr{A}_{\underline{q}}^{t c}$, such that $c_{q}\left(\tau_{b}^{\sharp}\right)=c_{q, \mathscr{A}_{q}^{t c}}^{*}$. Replace $\tau_{a}^{\sharp}$ with $\tau_{b}^{\sharp}$ in $\tau_{a}$. The resulting set of edges, say $\tau_{b}^{*}$, must be a $b$-tree. Observe that

$$
c_{q}\left(\tau_{b}^{*}\right)=c_{q}\left(\tau_{a}\right)-c_{q}\left(\tau_{a}^{\sharp}\right)+c_{q}\left(\tau_{b}^{\sharp}\right)<c_{q}\left(\tau_{a}\right) .
$$


The inequality comes from the fact that $a \notin M_{q}\left(\mathscr{A}_{\underline{q}}^{t c}\right)$. This contradicts that $c_{q}\left(\tau_{a}\right)=m_{q}^{*}$. "if" part: Suppose that $a \in M_{q}\left(\mathscr{A}_{\underline{q}}^{t c}\right)$ is not stochastically stable. Let $\tau_{a}^{\sharp}$ be an $a$-subtree over $\mathscr{A}_{q}^{t c}$, such that $c_{q}\left(\tau_{a}^{\sharp}\right)=c_{q, \mathscr{A}_{\underline{q}}^{t c}}^{*}$. Let $a^{\prime}$ be some stochastically stable policy with a minimum cost tree $\tau_{a^{\prime}}$. Lemmas B.1 and B.2 imply that $\tau_{a^{\prime}}$ has an $a^{\prime}$-subtree over $\mathscr{A}_{q}^{t c}$, say $\tau_{a^{\prime}}^{\sharp}$. Replace $\tau_{a^{\prime}}^{\sharp}$ with $\tau_{a}^{\sharp}$ in $\tau_{a^{\prime}}$. The resulting set of edges is an $a$-tree, say $\tau_{a}^{*}$. Observe that

$$
c_{q}\left(\tau_{a}^{*}\right)=c_{q}\left(\tau_{a^{\prime}}\right)-c_{q}\left(\tau_{a^{\prime}}^{\sharp}\right)+c_{q}\left(\tau_{a}^{\sharp}\right) \leq c_{q}\left(\tau_{a^{\prime}}\right)=m_{q}^{*} .
$$

Then, Theorem A.1 suggests that $a$ is stochastically stable, which is a contradiction.

For the multidimensional choice problems in Section 5, let $d\left(a, A^{\prime}\right)=\inf _{a^{\prime} \in A^{\prime}} d\left(a, a^{\prime}\right)$, for $a \in$ $\mathscr{A}^{0}$ and $A^{\prime} \subset \mathscr{A}^{0}$, that is, the distance between a point and a set. Let $\mathfrak{C}^{*, \sigma}$ denote a $\sigma$-neighborhood of $\mathfrak{C}^{*}$; that is, $\mathfrak{C}^{*}, \sigma=\left\{r \in \mathbb{R}^{h}: \inf _{r^{\prime} \in \mathfrak{C}^{*}} d\left(r, r^{\prime}\right)<\sigma\right\}$.

The following lemma describes the limiting properties of the min-max quota and the min-max set as the approximation $\delta$ goes to zero. The min-max set in the approximation space is included in the neighborhood of the min-max core for sufficiently small $\delta$.

Lemma B.3. (i) $\lim _{\delta \rightarrow 0} \bar{n}^{\delta}=\bar{n}$. (ii) Fix $\sigma>0 . \mathscr{A}^{m m, \delta} \subset \mathfrak{C}^{*, \sigma}$ for all sufficiently small $\delta$.

Proof of Lemma B.3. (i): By definition of $\bar{n}$, it holds that $n\left(a, a^{\prime}\right) \leq \bar{n}$, for $a \in \mathscr{A}^{\delta} \cap \mathfrak{C}^{*}$ and all $a^{\prime} \in \mathscr{A}^{\delta} \backslash\{a\}$. This implies that $\bar{n}^{\delta} \leq \bar{n}$. Suppose that $\lim _{\delta \rightarrow 0} \bar{n}^{\delta}<\bar{n}$. Choose $a \in \mathscr{A}^{\delta}$, such that $\lim _{\delta \rightarrow 0} n(a)=\lim _{\delta \rightarrow 0} \bar{n}^{\delta}<\bar{n}$. By the definition of $\bar{n}$, there exists $a^{\prime} \in \mathscr{A}^{0}$, such that $\mid i \in N$ : $d\left(s_{i}, a^{\prime}\right)<d\left(s_{i}, a\right) \mid \geq \bar{n}$. Choose $a^{\prime \prime} \in \mathscr{A}^{\delta}$, with $d\left(a^{\prime}, a^{\prime \prime}\right)<\delta$. By the continuity of $d$, we must have that $\left|i \in N: u_{i}\left(a^{\prime \prime}\right)>u_{i}(a)\right| \geq \bar{n}$, for all sufficiently small $\delta$. This contradicts that $\lim _{\delta \rightarrow 0} n(a)<\bar{n}$.

(ii): Because $\bar{n}^{\delta}$ takes only finite integers, (i) implies that $n(a)=\bar{n}^{\delta}=\bar{n}$, for all $a \in \mathscr{A}^{m m, \delta}$ and all sufficiently small $\delta$. Suppose that $\delta$ is small enough that $\bar{n}^{\delta}=\bar{n}$.

It suffices to show that $\mathscr{A}^{m m, \delta} \cap\left(\mathscr{A}^{0} \backslash \mathfrak{C}^{*, \sigma}\right)=\emptyset$. Choose $r \in \mathscr{A}^{0} \backslash \mathfrak{C}^{*, \sigma}$. Note that $d\left(r, \mathfrak{C}^{*}\right) \geq \sigma$. Because $r \notin \mathfrak{C}^{*}$, there exists some $r^{*} \in \mathscr{A}^{0}$, such that $\left|i \in N: u_{i}\left(r^{*}\right)>u_{i}(r)\right| \geq \bar{n}+1$. By the continuity of $d$, there exists $a^{\delta} \in \mathscr{A}^{\delta}$, with $d\left(r^{*}, a^{\delta}\right)<\delta$, such that $\left|i \in N: u_{i}\left(a^{\delta}\right)>u_{i}(r)\right| \geq$ $\bar{n}+1$, for all sufficiently small $\delta$. Because the choice of $r$ is arbitrary, this implies that, for all $r \in \mathscr{A}^{0} \backslash \mathfrak{C}^{*, \sigma}, r \notin \mathscr{A}^{m m, \delta}$, for all sufficiently small $\delta$.

In what follows, we assume that $\delta$ is sufficiently small such that $\bar{n}^{\delta}=\bar{n}$. Lemma B.4 below shows that any pair of policies can be connected via a sequence of pairwise voting under a $q$ majority if $q \leq \bar{n}$ and $\mathscr{A}^{0}$ is sufficiently large. McKelvey (1976) shows a similar result for an infinite state space and the simple majority rule. We prove the result for a finite state space, where opposing policies are selected only from $\mathscr{A}^{\delta}$, and extend it to a $q$-majority for all $q \leq \bar{n}$. 
Lemma B.4. For any $\rho>0$, let $B(\rho)=\left\{r \in \mathbb{R}^{h}:\|r\|<\rho\right\}$ and $B^{\delta}(\rho)=\left\{a \in \mathscr{A}^{\delta}:\|a\|<\rho\right\}$. Fix $\rho>0$ such that it is sufficiently large that $s_{i} \in B(\rho)$, for all $i \in N$. If $\mathscr{A}^{0}$ is sufficiently large that $B(5 \rho) \subset \mathscr{A}^{0}$, then for all $a_{1}, a_{L} \in B^{\delta}(\rho)$ and all sufficiently small $\delta$, there exists a sequence $\left\{a_{1}, a_{2}, \ldots, a_{L}\right\} \subset \mathscr{A}^{\delta}$, such that

$$
n\left(a_{j}, a_{j+1}\right) \geq \bar{n} \quad \forall j \in\{1, \ldots, L-1\} .
$$

Proof of Lemma B.4. The algorithm of McKelvey (1976, Theorem 2) can be extended to all quotas $q \leq \bar{n}$. The algorithm implies that for every $a_{k} \in \mathscr{A}^{\delta}$, there exist $c_{k}>0, r_{k+1} \in \mathscr{A}^{0}, y_{k} \in \mathbb{R}^{h}$, $\left\|y_{k}\right\|=1$, such that (i) $\left\|r_{k+1}\right\|^{2}-\left\|a_{k}\right\|^{2} \geq c_{k}^{2}$, (ii) $u_{j}\left(r_{k+1}\right)>u_{j}\left(a_{k}\right)$ if $s_{j}^{\prime} \cdot y_{k} \geq c_{k} / 2$, and (iii) $\mid\{j \in$ $\left.N: s_{j}^{\prime} \cdot y_{k} \geq c_{k}\right\} \mid \geq q$. Property (i) implies that $r_{k+1}$ is further from the origin than $a_{k}$ is. Properties (ii) and (iii) together imply that at least $q$ players prefer $r_{k+1}$ to $a_{k}$. Then, for all sufficiently small $\delta$, we can find $\hat{c}_{k} \in\left(0, c_{k}\right)$ and $a_{k+1} \in \mathscr{A}^{\delta}$, where $\left\|r_{k+1}\right\|-\left\|a_{k+1}\right\| \leq \delta$, such that $\left\|a_{k+1}\right\|^{2}-\left\|a_{k}\right\|^{2} \geq$ $\hat{c}_{k}^{2}$ and $u_{j}\left(a_{k+1}\right)>u_{j}\left(a_{k}\right)$ if $s_{j}^{\prime} \cdot y_{k} \geq \hat{c}_{k} / 2$. This implies that $a_{k+1}$ is preferred to $a_{k}$ by at least $q$ players. A successive application of the algorithm yields $a_{k}$ as far from the origin as required.

We show the upper bound of $\left\|r_{k+1}\right\|$ for given $a_{k}$. Recall that $r_{k+1}$ is preferred to $a_{k}$ by at least $q$ players. This implies that $r_{k+1} \in B(3 \rho)$ if $a_{k} \in B(\rho)$, and that $r_{k+1} \in B(5 \rho)$ if $a_{k} \in B(3 \rho)$. For the first case, observe that $r_{k+1}$ will be at least $2 \rho$ from $s_{i}$, for all $i \in N$, if $r_{k+1} \notin B(3 \rho)$. However, $a_{k} \in B(\rho)$ implies that $\left\|s_{i}-a_{k}\right\|<2 \rho$, for all $i \in N$. Then, for at least $q$ players to prefer $r_{k+1}$, it must be that $r_{k+1} \in B(3 \rho)$. Similarly, $r_{k+1}$ will be at least $4 \rho$ from all ideal points if $r_{k+1} \notin B(5 \rho)$, whereas $a_{k} \in B(3 \rho)$ implies that $\left\|s_{i}-a_{k}\right\|<4 \rho$, for all $i \in N$.

Finally, we show that the process can reach $a_{L} \in \mathscr{A}^{m m, \delta}$. Let $B^{*}=B(5 \rho) \backslash B(3 \rho)$; that is, the distance from any point in $B^{*}$ to any point in $B(\rho)$ is at least $2 \rho$. Because the algorithm yields $a_{k}$ as far as we need, we can pick a sequence $\left\{a_{1}, \ldots, a_{L-1}\right\}$, such that $a_{L-2} \in B(3 \rho)$ and $a_{L-1} \in B^{*}$. The proof is complete by observing that $n\left(a_{L-1}, a_{L}\right) \geq q$.

We next prove a result similar to Kramer (1977, Theorem 1'): $\bar{Q}(a)$ is the set of policies that maximizes the number of votes against policy $a$, i.e., $\bar{Q}(a)$ denotes the set of the most likely deviations from $a$. We first prove our version of Kramer (1977, Lemma 3).

Lemma B.5. Fix small $\delta>0$, such that $\bar{n}^{\delta}=\bar{n}$. Then, $d\left(a, \mathfrak{C}^{*}\right)>d\left(a^{\prime}, \mathfrak{C}^{*}\right)$, for $a \notin \mathscr{A}^{m m, \delta}$ and $a^{\prime} \in \bar{Q}(a)$.

Proof of Lemma B.5. Suppose that $a \notin \mathscr{A}^{m m, \delta}$ and $a^{\prime} \in \bar{Q}(a)$. Let $N\left(a, a^{\prime}\right)=J$. Let $\mathfrak{C}(J)$ denote the convex hull of the ideal points of the players in $J$. Because $a$ is not in the min-max set, $|J| \geq \bar{n}+1$. Define an open half space $V_{a^{\prime}}=\left\{x \in \mathbb{R}^{h}: d(x, a)>d\left(x, a^{\prime}\right)\right\}$. It must hold that $\mathfrak{C}(J) \subset V_{a^{\prime}}$. Otherwise, some player of $J$ must prefer $a$ to $a^{\prime}$, which contradicts the definition of $N\left(a, a^{\prime}\right)$. If there exists $b \in \mathfrak{C}^{*}$, such that $b \notin \mathfrak{C}(J)$, then some policy in $\mathfrak{C}(J)$ must be preferred to $b$ 
by all players in $J$, which contradicts that $b \in \mathfrak{C}^{*}$. This implies that $\mathfrak{C}^{*} \subseteq \mathfrak{C}(J) \subset V_{a^{\prime}}$. This proves the claim that $d\left(a, \mathfrak{C}^{*}\right)>d\left(a^{\prime}, \mathfrak{C}^{*}\right)$.

The next lemma is our version of Kramer (1977, Theorem 1').

Lemma B.6. For every $a_{1} \notin \mathscr{A}^{m m, \delta}$, there exists a sequence $\left\{a_{1}, a_{2}, \ldots, a_{L}\right\} \subset \mathscr{A}^{\delta}$ with $a_{L} \in$ $\mathscr{A}^{m m, \delta}$, such that $a_{i+1} \in \bar{Q}\left(a_{i}\right)$, for all $i=1, \ldots, L-1$.

Proof of Lemma B.6. Note that sequentially choosing $a_{i+1} \in \bar{Q}\left(a_{i}\right)$ must result in a cycle, owing to the finiteness of $\mathscr{A}^{\delta}$. Let $\left\{a_{1}, a_{2}, \ldots, a_{L}\right\}$ denote such a cyclic sequence of policies; that is, $a_{i+1} \in \bar{Q}\left(a_{i}\right)$, for all $i \in\{1, \ldots, L\}$, with a convention that $a_{L+1}=a_{1}$. We show that such a cycle must include $a_{i} \in \mathscr{A}^{m m, \delta}$ for some $i$.

By way of contradiction, suppose there exists a sequence $\left\{a_{1}, a_{2}, \ldots, a_{L}\right\}$, such that $a_{i} \notin \mathscr{A}^{m m, \delta}$, for all $i$. Then, Lemma B.5 implies that $d\left(a_{i}, \mathfrak{C}^{*}\right)>d\left(a_{i+1}, \mathfrak{C}^{*}\right)$, for all $i \in\{1, \ldots, L\}$; that is, the distance between $a_{i}$ and $\mathfrak{C}^{*}$ is strictly decreasing as the sequence $\left\{a_{1}, a_{2}, \ldots\right\}$ progresses. Because $d\left(a_{1}, \mathfrak{C}^{*}\right)>\ldots>d\left(a_{L+1}, \mathfrak{C}^{*}\right)$ implies that $a_{1} \neq a_{L+1}$, which contradicts that the sequence is cyclic.

Note that the distance increases $\left(d\left(a_{i}, \mathfrak{C}^{*}\right) \leq d\left(a_{i+1}, \mathfrak{C}^{*}\right)\right)$ only if $a_{i} \in \mathscr{A}^{m m, \delta}$. By sequentially choosing $a_{i+1} \in \bar{Q}\left(a_{i}\right)$, the process must reach some $a_{i} \in \mathscr{A}^{m m, \delta}$.

Proof of Theorem 2. (i) : Suppose that $q \leq \bar{n}$. It suffices to show that the unperturbed dynamic with state space $\mathscr{A}^{\delta}$ has a unique recurrent class that includes the open ball $B^{\delta}(\rho)$. Let $\mathfrak{C}(N)$ denote the convex hull of all players' ideal points. Lemma B.4 implies that all policies in $B^{\delta}(\rho)$ are connected via zero-cost transitions, for all sufficiently small $\delta$ and all sufficiently large $\mathscr{A}^{0}$. These policies must be in one recurrent class, say $A \subset \mathscr{A}^{\delta}$. Assume that $\mathscr{A}^{0}$ is sufficiently large that $\mathfrak{C}(N) \subseteq A$. We show that there is no recurrent class other than $A$. Choose $a^{\prime} \in \mathscr{A}^{\delta} \backslash A$. Let $a^{*} \in \operatorname{argmin}_{r \in \mathfrak{C}(N)} d\left(a^{\prime}, r\right)$. Observe that $u_{i}\left(a^{*}\right)>u_{i}\left(a^{\prime}\right)$, for all $i \in N$. By the continuity of $d$, for sufficiently small $\delta$, there exists $\hat{a}^{*} \in A$, with $d\left(a^{*}, \hat{a}^{*}\right)<\delta$, such that $u_{i}\left(\hat{a}^{*}\right)>u_{i}\left(a^{\prime}\right)$, for all $i \in N$. The cost of the transition from $a^{\prime}$ to $\hat{a}^{*}$ is zero. Because the cost must be positive for transitions between two recurrent classes, $a^{\prime}$ cannot be in any recurrent class. This proves that $A$ is the unique recurrent class.

(ii) : Suppose that $q>\bar{n}$. Assume sufficiently small $\delta$, such that $\bar{n}^{\delta}=\bar{n}$. By way of contradiction, assume there exists a stochastically stable policy $a_{1} \notin \mathscr{A}^{m m, \delta}$. Let $\tau_{1}$ denote the minimum cost spanning tree rooted at $a_{1}$. We show that the minimum cost spanning tree rooted at some $a_{L} \in$ $\mathscr{A}^{m m, \delta}$ has a strictly smaller cost than that of $a_{1}$.

Lemma B.6 implies that there exists a sequence $\left\{a_{1}, a_{2}, \ldots, a_{L}\right\} \subseteq \mathscr{A}^{\delta}$, with $a_{L} \in \mathscr{A}^{m m, \delta}$, such that $a_{i+1} \in \bar{Q}\left(a_{i}\right)$, for all $i \in\{1, \ldots, L-1\}$. Construct a path of edges $\left\{\left(a_{1}, a_{2}\right), \ldots,\left(a_{L-1}, a_{L}\right)\right\}$. 
Add these edges to $\tau_{1}$, replacing the existing edges exiting $a_{2}, \ldots, a_{L-1}$. Remove the edge exiting $a_{L}$. The resulting set of edges must be an $a_{L}$-tree, denoted as $\tau_{L}$. Then, observe that

$$
\begin{aligned}
c_{q}\left(\tau_{L}\right) & \leq c_{q}\left(\tau_{1}\right)+\sum_{i=1}^{L-1} \max \left\{q-n\left(a_{i}, a_{i+1}\right), 0\right\}-\sum_{i=2}^{L} \max \left\{q-\bar{n}\left(a_{i}\right), 0\right\} \\
& =c_{q}\left(\tau_{1}\right)+\max \left\{q-n\left(a_{1}, a_{2}\right), 0\right\}-\max \left\{q-\bar{n}\left(a_{L}\right), 0\right\} \\
& <c_{q}\left(\tau_{1}\right)=m_{q}^{*}
\end{aligned}
$$

The last term of the first inequality represents the cost reduction by removing the existing edges $a_{2}, \ldots, a_{L-1}$. The weak inequality holds because $\max \left\{q-\bar{n}\left(a_{i}\right), 0\right\}$ is the smallest possible cost of an edge exiting $a_{i}$. The second equality holds because $n\left(a_{i}, a_{i+1}\right)=\bar{n}\left(a_{i}\right)$, from the definition of the sequence $\left\{a_{1}, \ldots, a_{L}\right\}$. The last inequality holds because $a_{1} \notin \mathscr{A}^{m m, \delta}$ implies $n\left(a_{1}, a_{2}\right)>\bar{n}=$ $\bar{n}\left(a_{L}\right)$. Thus, $\tau_{L}$ has a strictly smaller cost than $\tau_{1}$, which contradicts that $a_{1}$ is stochastically stable. Therefore, no $a_{1} \notin \mathscr{A}^{m m, \delta}$ can be stochastically stable.

Proof of Theorem 3. Our setting for $q=n$ is similar to the unanimity game studied in Sawa (2014). The proof follows that of Proposition 4.6 in Sawa (2014). Using Eq. (4), define the cost of a tree $c_{q}(\cdot)$, cost of a policy $c_{q}^{*}(\cdot)$, minimum cost $m_{q}^{*}$, and set of policies with the minimum cost similarly to Eq. (A.4)-(A.6), respectively. As with Theorem A.1 for the BRM, a policy is stochastically stable under the logit choice if and only if it is in the set of policies with the minimum cost.

Observe that, for all $a, a^{\prime} \in \mathscr{A}$,

$$
\mathscr{P}(a)-\mathscr{P}\left(a^{\prime}\right)=\sum_{i \in N}\left(u_{i}(a)-u_{i}\left(a^{\prime}\right)\right)=c_{a a^{\prime}}^{n}-c_{a^{\prime} a}^{n}
$$

It suffices to show that for every $a_{1}$ and $a_{k}$ in $\mathscr{A}, \mathscr{P}\left(a_{1}\right) \geq \mathscr{P}\left(a_{k}\right)$ if and only if $c_{n}^{*}\left(a_{1}\right) \leq c_{n}^{*}\left(a_{k}\right)$. Let $\tau_{k}^{*}$ be an $a_{k}$-tree, such that $c_{n}\left(\tau_{k}^{*}\right)=c_{n}^{*}\left(a_{k}\right)$. Let $d=\left\{\left(a_{1}, a_{2}\right), \ldots,\left(a_{k-1}, a_{k}\right)\right\}$ be a path from $a_{1}$ to $a_{k}$ in the tree $\tau_{k}^{*}$. We construct an $a_{1}$-tree, denoted as $\tau_{1}$, from $\tau_{k}^{*}$ by reversing the directions of all edges on the path $d$, and keeping all other edges in $\tau_{k}^{*}$. Formally, let $\tau_{1}$ be such that

$$
\tau_{1} \ni \begin{cases}\left(a^{\prime}, a^{\prime \prime}\right) & \text { if }\left(a^{\prime}, a^{\prime \prime}\right) \in \tau_{k}^{*} \backslash d \\ \left(a^{\prime \prime}, a^{\prime}\right) & \text { if }\left(a^{\prime}, a^{\prime \prime}\right) \in d\end{cases}
$$

Observe that

$$
c_{n}^{*}\left(a_{1}\right) \leq c_{n}\left(\tau_{1}\right)=c_{n}\left(\tau_{k}^{*}\right)+\sum_{\left(a_{i}, a_{i+1}\right) \in d}\left(c_{a_{i+1} a_{i}}^{n}-c_{a_{i} a_{i+1}}^{n}\right)=c_{n}^{*}\left(a_{k}\right)+\mathscr{P}\left(a_{k}\right)-\mathscr{P}\left(a_{1}\right)
$$

We use Eq. (B.2) in the second equality. The above equation shows that $\mathscr{P}\left(a_{1}\right) \geq \mathscr{P}\left(a_{k}\right)$ implies 
$c_{n}^{*}\left(a_{1}\right) \leq c_{n}^{*}\left(a_{k}\right)$.

\section{References}

Acemoglu, D., Egorov, G., and Sonin, K. (2011). Political model of social evolution. Proceedings of the National Academy of Sciences, 108(Supplement_4):21292-21296.

Acemoglu, D., Egorov, G., and Sonin, K. (2012). Dynamics and stability of constitutions, coalitions, and clubs. American Economic Review, 102:1446-1476.

Agastya, M. (1999). Perturbed adaptive dynamics in coalition form games. Journal of Economic Theory, 89:207-233.

Aghion, P., Alesina, A., and Trebbi, F. (2004). Endogenous political institutions. Quarterly Journal of Economics, 119:565-611.

Alós-Ferrer, C. and Netzer, N. (2010). The logit-response dynamics. Games and Economic Behavior, 68:413-427.

Anesi, V. (2010). Noncooperative foundations of stable sets in voting games. Games and Economic Behavior, 70(2):pp.488-493.

Anesi, V. and Seidmann, D. J. (2015). Bargaining in standing committees with an endogenous default. Review of Economic Studies, 82:pp.825-867.

Arnold, T. and Schwalbe, U. (2002). Dynamic coalition formation and the core. Journal of Economic Behavior \& Organization, 49:363-380.

Banks, J. S. and Duggan, J. (2005). Probabilistic Voting in the Spatial Model of Elections: The Theory of Office-motivated Candidates, pages 15-56. Springer Berlin Heidelberg, Berlin, Heidelberg.

Barbera, S. and Jackson, M. O. (2004). Choosing how to choose: Self-stable majority rules and constitutions. Quarterly Journal of Economics, 119:1011-1048.

Baron, D. P. (1996). A dynamic theory of collective goods programs. American Political Science Review, 90:316-330.

Baron, D. P. and Ferejohn, J. A. (1989). Bargaining in legislatures. American Political Science Review, 83:1181-1206. 
Battaglini, M. and Coate, S. (2007). Inefficiency in legislative policymaking: A dynamic analysis. American Economic Review, 97(1):pp.118-149.

Belloc, M. and Bowles, S. (2017). Persistence and change in culture and institutions under autarchy, trade, and factor mobility. American Economic Journal: Microeconomics, 9(4):245276.

Bendor, J., Diermeier, D., and Ting, M. (2003). A behavioral model of turnout. American Political Science Review, 97(02):261-280.

Bilancini, E., Boncinelli, L., and Newton, J. (2020). Evolution and rawlsian social choice in matching. Games and Economic Behavior, 123:68-80.

Binmore, K. G. (1994). Game Theory and the Social Contract. MIT Press, Cambridge, Mass.

Binmore, K. G. (2005). Natural Justice. Oxford University Press, New York.

Black, D. (1948). On the rationale of group decision-making. Journal of Political Economy, $56: 23-34$.

Blume, L. (1993). The statistical mechanics of strategic interaction. Games and Economic Behavior, 5:387-424.

Boncinelli, L. and Pin, P. (2018). The stochastic stability of decentralized matching on a graph. Games and Economic Behavior, 108:239-244.

Borda, Jean Charles de. (1781). Mémoire sur les elections au scrutin. In Histoire de L'Academie Royale des Sciences.

Caplin, A. and Nalebuff, B. (1988). On 64\%-majority rule. Econometrica, 56:787-814.

Condorcet, Marquis de. (1785). Essai sur l'application de l'analyse à la probabilité des décisions rendues à la probabilith des voix. Paris: De l'imprimerie royale.

Conley, J. P., Toossi, A., and Wooders, M. (2006). Memetics and voting: how nature may make us public spirited. International Journal of Game Theory, 35(1):71-90.

Coughlin, P. J. (1992). Probabilistic Voting Theory. Cambridge University Press.

Davis, O. A., DeGroot, M. H., and Hinich, M. J. (1972). Social preference orderings and majority rule. Econometrica, 40:147-157. 
Demuynck, T., Herings, P. J.-J., Saulle, R. D., and Seel, C. (2019). The myopic stable set for social environments. Econometrica, 87(1):111-138.

Diermeier, D., Egorov, G., and Sonin, K. (2017). Political economy of redistribution. Econometrica, 85(3):pp.851-870.

Duggan, J. and Kalandrakis, T. (2012). Dynamic legislative policy making. Journal of Economic Theory, 147:1653-1688.

Foster, D. P. and Young, H. P. (1990). Stochastic evolutionary game dynamics. Theoretical Population Biology, 38:219-232.

Fowler, J. H. (2006). Habitual voting and behavioral turnout. The Journal of Politics, 68(2):335344.

Golub, B. and Jackson, M. O. (2010). Naïve learning in social networks and the wisdom of crowds. American Economic Journal: Microeconomics, 2(1):112-149.

Gomes, A. and Jehiel, P. (2005). Dynamic processes of social and economic interactions: On the persistence of inefficiencies. Journal of Political Economy, 113:626-667.

Grabisch, M. and Rusinowska, A. (2020). A survey on nonstrategic models of opinion dynamics. Games, 11(4):65.

Hwang, S.-H., Lim, W., Neary, P., and Newton, J. (2018). Conventional contracts, intentional behavior and logit choice: Equality without symmetry. Games and Economic Behavior, 110:273294.

Jackson, M. O. and Watts, A. (2002). The evolution of social and economic networks. Journal of Economic Theory, 106:265-295.

Kalandrakis, A. (2004). A three-player dynamic majoritarian bargaining game. Journal of Economic Theory, 116:294-322.

Kanazawa, S. (1998). A possible solution to the paradox of voter turnout. The Journal of Politics, 60(4):974-995.

Kandori, M., Mailath, G. J., and Rob, R. (1993). Learning, mutation, and long run equilibria in games. Econometrica, 61:29-56.

Kandori, M., Serrano, R., and Volij, O. (2008). Decentralized trade, random utility and the evolution of social welfare. Journal of Economic Theory, 140:328-338. 
Klaus, B., Klijn, F., and Walzl, M. (2010). Stochastic stability for roommate markets. Journal of Economic Theory, 145(6):2218 - 2240.

Kramer, G. H. (1977). A dynamical model of political equilibrium. Journal of Economic Theory, $16: 310-334$.

Lagunoff, R. D. and Matsui, A. (1995). Evolution in mechanisms for public projects. Economic Theory, 6(2):195-223.

Landi, M. and Sodini, M. (2012). An evolutionary analysis of turnout with conformist citizens. Journal of Economic Dynamics and Control, 36(10):1431-1447.

Lim, W. and Neary, P. R. (2016). An experimental investigation of stochastic adjustment dynamics. Games and Economic Behavior, 100:208-219.

Mäs, M. and Nax, H. H. (2016). A behavioral study of "noise" in coordination games. Journal of Economic Theory, 162:195-208.

McKelvey, R. D. (1976). Intransitivities in multidimensional voting models and some implications for agenda control. Journal of Economic Theory, 12:472-482.

McKelvey, R. D. (1979). General conditions for global intransitivities in formal voting models. Econometrica, 47:1085-1112.

McKelvey, R. D. and Patty, J. W. (2006). A theory of voting in large elections. Games and Economic Behavior, 57(1):155-180.

Miller, N. R. (1977). Graph-theoretical approaches to the theory of voting. American Journal of Political Science, 21:769-803.

Nax, H. H. and Pradelski, B. S. R. (2015). Evolutionary dynamics and equitable core selection in assignment games. International Journal of Game Theory, 44:903-932.

Nax, H. H. and Rigos, A. (2016). Assortativity evolving from social dilemmas. Journal of Theoretical Biology, 395:194-203.

Newton, J. (2012). Coalitional stochastic stability. Games and Economic Behavior, 75:842—-854.

Newton, J. (2018). Evolutionary game theory: A renaissance. Games, 9(2):31.

Newton, J. and Sawa, R. (2015). A one-shot deviation principle for stability in matching problems. Journal of Economic Theory, 157:1-27. 
Nunnari, S. (2021). Dynamic legislative bargaining with veto power: Theory and experiments. Games and Economic Behavior, 126:186-230.

Penn, E. M. (2009). A model of farsighted voting. American Journal of Political Science, 53:3654.

Plott, C. R. (1967). A notion of equilibrium and its possibility under majority rule. American Economic Review, 57:787-806.

Riker, W. H. (1980). Implications from the disequilibrium of majority rule for the study of institutions. American Political Science Review, 74:432-446.

Sandholm, W. H. (2010). Population Games and Evolutionary Dynamics. MIT Press, first edition.

Sawa, R. (2014). Coalitional stochastic stability in games, networks and markets. Games and Economic Behavior, 88:90-111.

Schofield, N. (2005). Local Political Equilibria, pages 57-91. Springer Berlin Heidelberg, Berlin, Heidelberg.

Simpson, P. B. (1969). On defining areas of voter choice: Professor tullock on stable voting. Quarterly Journal of Economics, 83:478-490.

Skyrms, B. (1996). Evolution of the Social Contract. Cambridge University Press, Cambridge New York.

Tullock, G. (1967). The general irrelevance of the general impossibility theorem. Quarterly Journal of Economics, 81:256-270.

Wu, J. (2016). Evolving assortativity and social conventions. Economics Bulletin, 36(2):936-941.

$\mathrm{Wu}$, J. (2017). Political institutions and the evolution of character traits. Games and Economic Behavior, 106:260-276.

Young, H. P. (1993). The evolution of conventions. Econometrica, 61:57-84.

Young, H. P. (1998). Conventional contracts. Review of Economic Studies, 65(4):773-792. 